Research Article

\title{
Performance Comparison of Bow and Stern Rudder for High-Speed Supercavitating Vehicles
}

\author{
Chuang Huang (D), Kai Luo, Kan Qin, Daijin Li iD, and Jianjun Dang \\ School of Marine Science and Technology, Northwestern Polytechnical University, Xi'an 710072, China \\ Correspondence should be addressed to Chuang Huang; huangchuang@nwpu.edu.cn
}

Received 21 November 2019; Revised 15 February 2020; Accepted 21 May 2020; Published 15 June 2020

Academic Editor: Francisco J. Montáns

Copyright (c) 2020 Chuang Huang et al. This is an open access article distributed under the Creative Commons Attribution License, which permits unrestricted use, distribution, and reproduction in any medium, provided the original work is properly cited.

\begin{abstract}
To predict the hydrodynamic characteristics and supercavity shape of supercavitation flows, the numerical model including VOF, cavitation model, and turbulence models is presented and validated by a well-established empirical correlation. The numerical method is then employed to simulate the high-speed supercavitating vehicles with two different types of control surfaces: bow rudders and stern rudders. The hydrodynamic characteristics and influences on the supercavity are compared. By contrast with the stern rudder, the bow rudder with the same wetted area is capable of generating a larger control force and moment. Also, the bow rudder introduces a considerable deformation to the forepart of the supercavity, while the stern rudder provides a negligible influence on the supercavity before it. In addition, the bow rudder is fully wetted, and the lift force only changes with the rudder angle. However, the stern rudder is partly wetted; the lift force is not only determined by the rudder angle but also related to the actual wetted status.
\end{abstract}

\section{Introduction}

High-speed supercavitating vehicles (HSSVs) are surrounded by supercavities and receive a very small skin friction drag as flying in air [1]. Therefore, the sailing drag of HSSVs is largely reduced. Moreover, the drag reduction is further enhanced with the decrease of cavitation number [2]. Then, the speed of HSSVs can be largely increased with respect to conventional underwater vehicles [3]. By employing this method, the large-sized and medium-sized underwater vehicles can achieve a speed of 200-400 kts, and supercavitating projectiles can even fly at a speed faster than the sound speed underwater $[1,4]$. However, the hydrodynamic characteristics and control strategy of HSSVs are largely different from the conventional underwater vehicles because of the existence of the supercavity and need to be further investigated [5].

In 1942, Loytsyansky successfully reduced the drag force of a cylindrical model in the water tunnel by generating the ventilated supercavitation for the first time [6]. Since then, attentions have been increasing on supercavitating flows.
The fundamental problems such as the generation mechanism, evolution law, and description of supercavity shape have been sufficiently studied $[7,8]$. To enable a self-guided and well-controlled HSSV, challenges including the hydrodynamic characteristics, kinetics modeling, and sailing control still need to be overcome.

The precise description of hydrodynamic characteristics is the prerequisite of establishing the kinetics model of HSSVs $[9,10]$. May [11] initially develops the empirical correlations to predict the hydrodynamic characteristics of the disk and cone cavitators in the high-speed water-entry process. Then, based on a series of water tunnel experiments, the relevant correlations are also amended and validated by Mokhtarzadeh et al. to calculate the lift and drag force of disk and cone cavitators in supercavitation flows [12]. The hydrodynamic forces of HSSVs only act on the wetted zone because of flying in the supercavity [13]. The cavitator continuously touches water and receives the lift force and drag force, and the forces exactly act at the nose of the HSSV. Thus, the other wetted parts, such as the hulls and rudders, are essential to maintain the force and moment balances of 
the HSSV during the directed and maneuvering movements $[14,15]$. The hydrodynamic force acting on the wetted hulls of the HSSV by puncturing the supercavity is also referred to as a planing force and directly related to the kinetic characteristics. The planing force is codetermined by the relative position between the supercavity and the hull of the HSSV and also affected by the size and shape of the supercavity [16]. Vasin and Paryshev [17] and Yen et al. [18] theoretically and experimentally investigated the hydrodynamic characteristics of HSSVs, found the strong nonlinearity existing in the planing force, and presented an empirical correlation to forecast it. Nguyen and Balachandran [19] further established the kinetics model of HSSVs by taking the nonlinear planing force into account and performed mathematical simulations and theoretical analyses. Then, they concluded that the movement of the HSSV is controllable, but the motion stability is closely related to the layout scheme and control strategy. The rudders are very crucial for the control of HSSVs and are traditionally arranged at the tail, for example, the "Shkval" designed by Russia. Based on this layout scheme, Li et al. study the kinetic characteristics and the motion stability of the directed HSSV by the lake-bed experiment [20]. Then, the variable splitting method is presented to regulate the HSSV in the maneuvering condition [21]. Luo et al. [22] presented a new layout scheme of HSSVs by moving the rudders to the nose and concluded that the HSSV controlled by the rudder from nose can achieve a smaller turning radius during the maneuvering motion by contrast with the traditional layout scheme.

As the control surface of HSSVs, the rudder plays an important role in the regulation of the posture and motion. Then, the wedge-shaped rudder, especially one with a wedge angle of $24^{\circ}$, is most widely used because of the good hydrodynamic performance in the supercavitation flow field $[10,20]$. The rudder provides lift force, also named as control force, by extending to the outside of the supercavity, which simultaneously causes the supercavity deformation and the change of the hydrodynamic characteristics of the HSSV [23]. Moreover, cavitation may suddenly occur at the suction surface of the wedge-shaped rudder with the increase of the rudder angle, and this would result in a discontinuity to the lift coefficients [24, 25]. Although some results about the wedge-shaped rudder operating in the supercavitation flow are obtained, most of the experiments are carried out in water tunnels and the operation conditions are far different from those of the actual HSSV. Further, the differences between the rudders mounted at the tail and nose are not detailed. In the layout scheme of HSSVs, the rudder mounted at tail is called stern rudder (SR), and that at nose is called bow rudder (BR). This paper aims to study the supercavity deformations induced by BRs and SRs and their hydrodynamic characteristics, respectively, and is organized as follows. Firstly, the geometries of the BR model and SR model are defined. Then, the computational model for supercavitation flows and validation cases are described. Next, the hydrodynamic characteristics of the BR and SR, as well as their respective influences on the supercavity, are calculated. Finally, the performances between the BR and SR are compared.

\section{Problem Description}

HSSVs are composed of the cavitators, fore-conic sections, cylindrical sections, rudders, and engine nozzles. Two layout schemes are typically employed by HSSV s and characterized by different kinds of control surfaces $[20,22]$. They are the BR layout scheme and SR layout scheme and are shown in Figures 1(a) and 1(b), respectively. The BRs and SRs are both arranged in the cruciform configuration as shown in Figure 2 and installed at the cone cavitator (Figure 2(a)) and the tail of the cylindrical section (Figure 2(b)) individually. It is shown in Figure 2(a) that the key parameters of the BR include the length of the chord and maximum span and $\alpha_{\mathrm{B}}$. In addition, a small hole is designed in the cone cavitator and used as the water inlet of the water-ramjet engine. Thus, the cone cavitator looks like a frustum.

The SR model is taken from a practical HSSV [20] and its geometric parameters are given in Table 1 . By referring to the parameters listed in Table 1, the BR model is designed. To have enough space installing the BRs and relevant structures, the disk cavitator of the SR model is first replaced by a cone cavitator.

The shape and dimensions of a supercavity are essentially determined by the cavitator drag and cavitation number and scarcely related to the concrete forms of the cavitator [11]. Namely, a cone cavitator and a disk cavitator suffering the identical drag can generate roughly the same supercavities when operating under the same conditions. Taking the base area of the cavitator as a reference, the drag coefficients of the disk and cone cavitators in the zero-attack angle condition can be calculated by [26]

$$
\begin{aligned}
C_{\mathrm{d}} & =C_{\mathrm{d} 0}+(0.524+0.672 \alpha) \sigma, \\
C_{\mathrm{d} 0} & =0.5+1.81(\alpha-0.25)-2(\alpha-0.25)^{2},
\end{aligned}
$$

where $C_{\mathrm{d} 0}$ is the drag coefficient when the cavitation number equals zero, and formula (2) is applicable for $1 / 12 \leq \alpha \leq 1 / 2$. $C_{\mathrm{d}}$ is the drag coefficient when the cavitation number is equal to $\sigma$ and $\pi \alpha$ is the semicone angle of the cavitator. In addition, $\alpha$ is 0.167 for a $60^{\circ}$ cone cavitator and is 0.5 for a disk cavitator.

According to formulas (1) and (2), the drag coefficient of the disk cavitator of the SR model is 0.847 in the design condition, and the drag coefficient of a cone cavitator can be calculated if the cone angle is known. Herein, a $60^{\circ}$ cone is assumed to be the cavitator of the BR model, and its drag coefficient is 0.350 in the design condition. Therefore, considering the disk cavitator has a diameter of $42 \mathrm{~mm}$; the equivalent cone cavitator should have a base diameter of $65.3 \mathrm{~mm}$. The geometric parameters of the cone cavitator are listed in Table 2.

The disk cavitator of the SR model is replaced by the equivalent cone cavitator by the above steps. Next, the SR is replaced by a BR that can produce the approximate same control moment as the SR when operating at the maximum rudder angle. After receiving the maneuvering command, the rudder deflects to provide an unbalanced moment to turning the HSSV. Resultantly, a deflection between the axes 


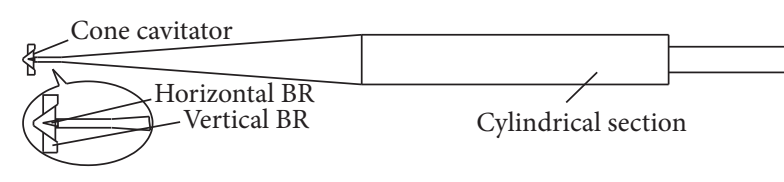

(a)

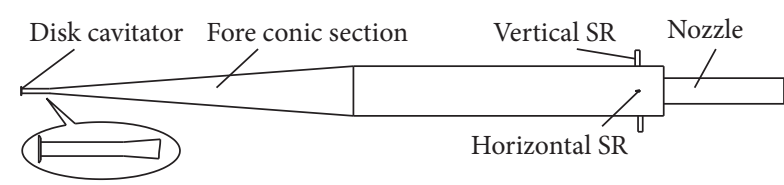

(b)

Figure 1: Two different layout schemes of HSSVs. (a) BR layout scheme. (b) SR layout scheme.

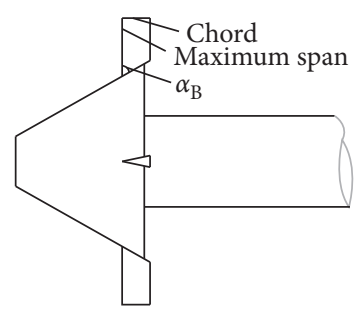

(a)

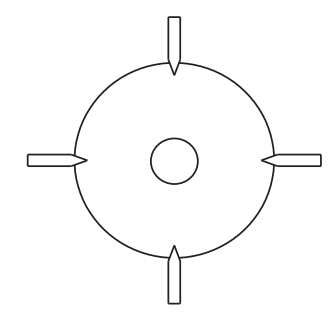

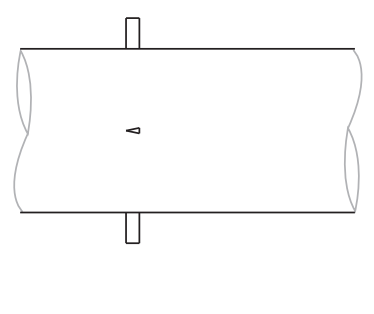

(b)

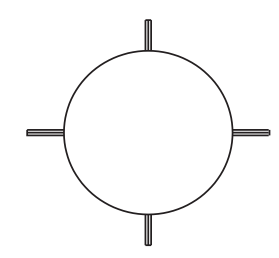

FIGURE 2: Configuration and installing position of BRs and SRs. (a) Configuration and installment of BRs. (b) Configuration and installment of SRs.

TABle 1: Design parameters of the SR model.

\begin{tabular}{lc}
\hline Items & Values \\
\hline Full length & $3200 \mathrm{~mm}$ \\
Distance from SR to cavitator & $2630 \mathrm{~mm}$ \\
Distance from mass center to cavitator & $1750 \mathrm{~mm}$ \\
Length of fore-conic section & $1400 \mathrm{~mm}$ \\
Length of cylindrical section & $1300 \mathrm{~mm}$ \\
Design speed & $100 \mathrm{~m} / \mathrm{s}$ \\
Length of nozzle & $510 \mathrm{~mm}$ \\
Maximum diameter & $210 \mathrm{~mm}$ \\
Span of SR & $46 \mathrm{~mm}$ \\
Diameter of disk cavitator & $42 \mathrm{~mm}$ \\
Chord of SR & $17.5 \mathrm{~mm}$ \\
Design cavitation number & 0.023 \\
\hline
\end{tabular}

TABLE 2: Geometric parameters of the BR model.

\begin{tabular}{lc}
\hline Items & Values \\
\hline Conic angle of cavitator & $60^{\circ}$ \\
Diameter of cone cavitator & $65.3 \mathrm{~mm}$ \\
Maximum span of BR & $20 \mathrm{~mm}$ \\
Chord of BR & $10 \mathrm{~mm}$ \\
\hline
\end{tabular}

of the HSSV and supercavity is formed, which causes the tail of the HSSV to touch water. Then, the planing force and moment acting on the hull are produced. During the turning condition, the planing moment is balanced by the control force provided by the rudder, and the sum of the planing force and control force serves as the centripetal force to turn the HSSV. Considering that the planing force is far larger than the control force, the maneuverability is mainly determined by the magnitude of the planing force. Then, the maximum planing force and moment of the BR layout scheme should be roughly equal to those of the SR layout scheme because of the same hulls and the approximate supercavities. Given that the distance from the BR to the mass center of the HSSV is about two times longer than that of the SR as listed in Table 1, the required control force of the BR to balance the planing moment in ultimate condition is just about half that of the SR. The hydrodynamic characteristics of the SR and BR and the effective wetted area of the $\mathrm{BR}$ can be achieved by numerical computations, and the area of the BR is subsequently calculated. According to the hydrodynamic characteristics of the SR and BR and the effective wetted area of the BR, the needed area of the BR is then achieved. Given that the BR is a right trapezoid having an acute angle $\alpha_{\mathrm{B}}$ that is equal to the complementary angle of the half cone angle of the cone cavitator, the maximum span can be preliminarily obtained by selecting a chord length, for example, $10 \mathrm{~mm}$. However, the hydrodynamic characteristics of the BR are related to the aspect ratio. The modified geometrical parameters of the BR model can be modified iteratively as shown in the flowchart in Figure 3.

The finally obtained geometric parameters of the BR model are listed in Table 2.

\section{Numerical Model}

3.1. Governing Equations. The governing equations for supercavitation flows include the incompressible continuity and momentum equations, while the energy equation is not considered. The thermodynamic properties at $25^{\circ} \mathrm{C}$ are $\rho_{1}=997.0 \mathrm{~kg} / \mathrm{m}^{3}$ and $\mu_{1}=8.9 \times 10^{-4} \mathrm{~Pa} \quad \mathrm{~s}$ and $\rho_{\mathrm{v}}=2.3 \times 10^{-2} \mathrm{~kg} / \mathrm{m}^{3}$ and $\mu_{\mathrm{v}}=9.9 \times 10^{-6} \mathrm{~Pa}$ s. Here, the subscripts 1 and $\mathrm{v}$ denote the liquid phase and vapor phase, respectively. The supercavitation flow field can be simulated by using the volume of fraction (VOF) model [27]. The phase change is treated as the mass transfer between the liquid and vapor phases and is mathematically described by the Schnerr and Sauer cavitation model. The realizable $k$-epsilon 


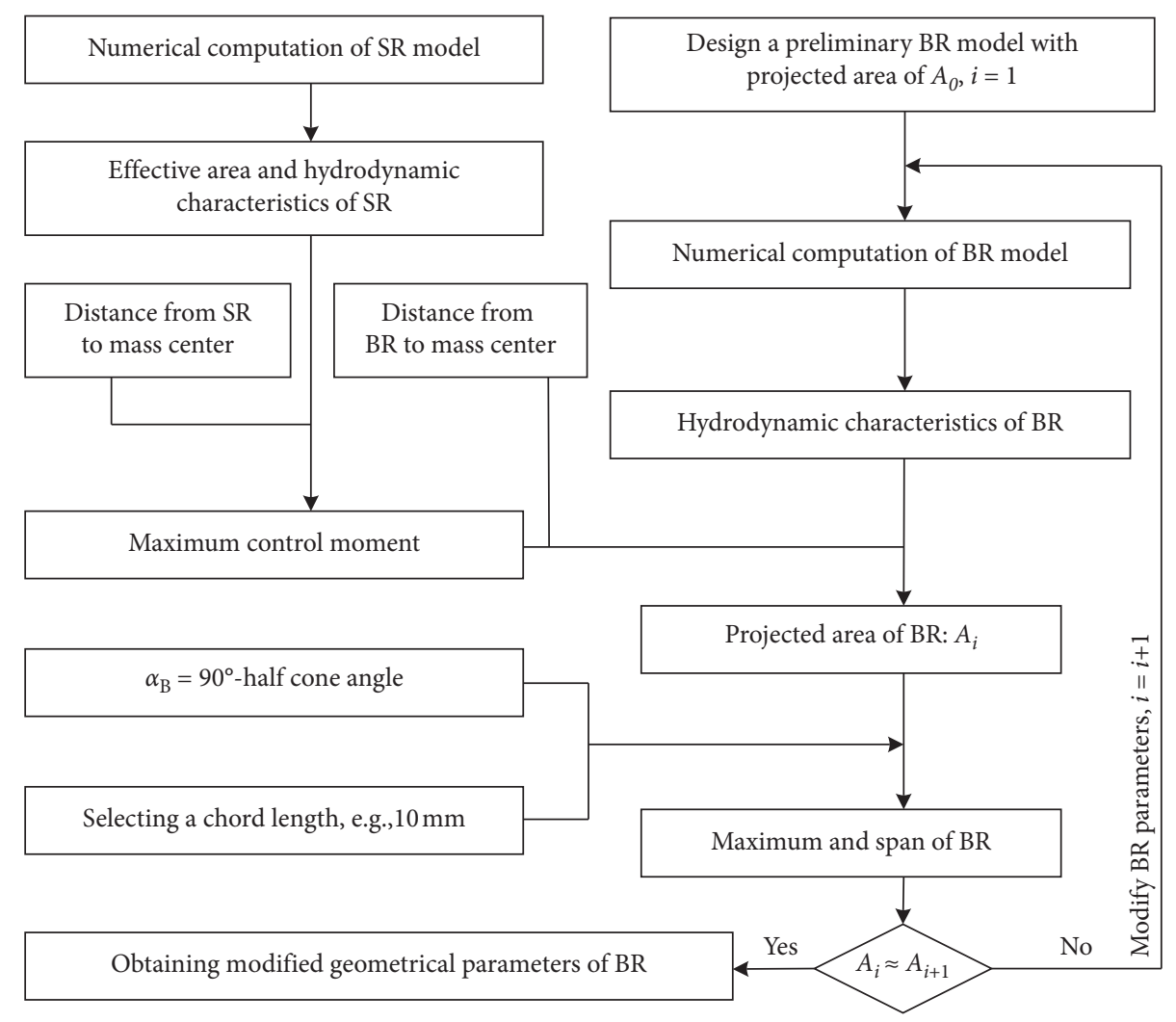

FIGURE 3: Flowchart to determine geometrical parameters of BRs.

turbulence model and the scale wall function are utilized to close the time-averaged conservation equations.

The governing continuity equation for a homogeneous multiphase flow is written as

$$
\frac{\partial}{\partial t}\left(\rho_{\mathrm{m}}\right)+\nabla\left(\rho_{\mathrm{m}} \mathbf{u}\right)=0
$$

The governing momentum equation for a homogeneous multiphase flow is expressed as

$$
\frac{\partial}{\partial t}\left(\rho_{\mathrm{m}} \mathbf{u u}\right)+\nabla \cdot\left(\rho_{\mathrm{m}} \mathbf{u u}\right)=-\nabla p+\nabla \cdot\left[\mu_{\mathrm{m}}\left(\nabla \mathbf{u}+\nabla \mathbf{u}^{\mathrm{T}}\right)\right] .
$$

The volume fraction of vapor phase is given as

$$
\nabla \cdot\left(\alpha_{\mathrm{v}} \rho_{\mathrm{v}} \mathbf{u}\right)=R_{\mathrm{e}}-R_{\mathrm{c}},
$$

where $\mathbf{u}$ is the velocity vector of the mixture phase; $p, \rho, \alpha$, and $\mu$ denote the pressure, density, volume fraction, and viscosity, respectively; subscripts $\mathrm{m}$ and $\mathrm{v}$ represent the mixture phase and the vapor phase, individually; and $R_{\mathrm{e}}$ and $R_{\mathrm{c}}$ are the evaporation rate of liquid phase and condensation rate of vapor phase, separately.

The density and viscosity of the mixture phase are expressed as

$$
\begin{aligned}
& \rho_{\mathrm{m}}=\alpha_{\mathrm{v}} \rho_{\mathrm{v}}+\left(1-\alpha_{\mathrm{v}}\right) \rho_{\mathrm{l}}, \\
& \mu_{\mathrm{m}}=\alpha_{\mathrm{v}} \mu_{\mathrm{v}}+\left(1-\alpha_{\mathrm{v}}\right) \mu_{\mathrm{w}} .
\end{aligned}
$$

According to Schnerr-Sauer cavitation model [28] the evaporation rate $R_{\mathrm{e}}$ and the condensation rate $R_{\mathrm{c}}$ are determined as follows.

For the condition $p \leq p_{\mathrm{B}}, R_{\mathrm{e}}$ is

$$
R_{\mathrm{e}}=F_{\text {evap }} \frac{\rho_{\mathrm{v}} \rho_{\mathrm{l}}}{\rho_{\mathrm{m}}} \frac{3}{R_{\mathrm{B}}} \alpha_{\mathrm{v}}\left(1-\alpha_{\mathrm{v}}\right) \sqrt{\frac{2}{3} \frac{\left(p_{\mathrm{B}}-p\right)}{\rho_{\mathrm{l}}}} .
$$

For the condition $p>p_{\mathrm{B}}, R_{\mathrm{c}}$ is

$$
R_{\mathrm{c}}=F_{\text {cond }} \frac{\rho_{\mathrm{v}} \rho_{1}}{\rho_{\mathrm{m}}} \frac{3}{\mathfrak{R}_{\mathrm{B}}} \alpha_{\mathrm{v}}\left(1-\alpha_{\mathrm{v}}\right) \sqrt{\frac{2}{\frac{\left(p-p_{\mathrm{B}}\right)}{\rho_{\mathrm{l}}}}},
$$

where $F_{\text {evap }}$ and $F_{\text {cond }}$ are the empirical calibration coefficients of evaporation and condensation and take the values of 1.0 and 0.2 , respectively. $p_{\mathrm{B}}$ is the internal static pressure of supercavities and $\mathfrak{R}_{\mathrm{B}}$ is the radius of bubbles and is defined as

$$
\mathfrak{R}_{\mathrm{B}}=\left[\frac{3 \alpha_{\mathrm{v}}}{4 \pi n_{\mathrm{b}}\left(1-\alpha_{\mathrm{v}}\right)}\right]^{1 / 3}
$$

where $n_{\mathrm{b}}$ is the number of bubbles in a unit volume of liquid and is set to be $1 \times 10^{11} \mathrm{~m}^{-3}$.

By neglecting the effect of the body force and compressibility of the fluid, the realizable $k$-epsilon turbulence model can be simplified as 


$$
\begin{aligned}
\frac{\partial\left(\rho_{\mathrm{m}} k\right)}{\partial t}+\frac{\partial\left(\rho_{\mathrm{m}} k u_{j}\right)}{\partial x_{j}}= & \frac{\partial}{\partial x_{j}}\left[\left(\mu_{\mathrm{m}}+\frac{\mu_{\mathrm{t}}}{\sigma_{\mathrm{k}}}\right) \frac{\partial k}{\partial x_{j}}\right]+G_{\mathrm{k}}-\rho \varepsilon_{\mathrm{m}} \\
\frac{\partial\left(\rho_{\mathrm{m}} \varepsilon\right)}{\partial t}+\frac{\partial\left(\rho_{\mathrm{m}} \varepsilon u_{j}\right)}{\partial x_{j}}= & \frac{\partial}{\partial x_{j}}\left[\left(\mu_{\mathrm{m}}+\frac{\mu_{\mathrm{t}}}{\sigma_{\varepsilon}}\right) \frac{\partial \varepsilon}{\partial x_{j}}\right] \\
& +\rho_{\mathrm{m}} C_{1} S \varepsilon-\frac{\rho_{\mathrm{m}} C_{2} \varepsilon^{2}}{k+\sqrt{\nu \varepsilon}}
\end{aligned}
$$

where $C_{1}=\max [0.43, \eta / \eta+5]$ and $\eta=S k / \varepsilon ; G_{\mathrm{k}}$ represents the generation of turbulence kinetic energy due to mean velocity gradient; $C_{2}$ is a constant that is equal to $1.9 ; \sigma_{\mathrm{k}}=1.0$ and $\sigma_{\varepsilon}=1.2$ are the turbulent Prandtl numbers of $k$ and $\varepsilon$, respectively; and $\mu_{\mathrm{t}}$ denotes the eddy viscosity.

The realizable $k$-epsilon turbulence model is suitable to predict the fully developed turbulent flow and is not good at predicting the flow status in the boundary layer. Thus, the near-wall treatment is essential to improve the computational precision of turbulences. The scale wall function is an improved empirical model to describe the velocity distribution in the boundary layer and can ensure the high calculating stability and precision even for complex flow by setting a limiter to $y^{*}$. The scale wall function is expressed as

$$
\left\{\begin{array}{l}
U^{*}=\frac{1}{\kappa} \ln \left(E y^{*}\right), \\
U^{*} \equiv \frac{U_{\mathrm{p}} C_{\mu}^{1 / 4} k_{\mathrm{p}}^{1 / 2}}{\tau_{\mathrm{w}} / \rho}, \\
y^{*}=\frac{\rho_{\mathrm{m}} C_{\mu}^{1 / 4} k_{\mathrm{p}}^{1 / 2} y_{\mathrm{p}}}{\mu_{\mathrm{m}}}, \quad y^{*} \geq 11, \\
y^{*}=\max \left(y^{*}, y_{\lim }^{*}\right), \quad y^{*}<11 .
\end{array}\right.
$$

where $U^{*}$ is the nondimensional velocity, $y^{*}$ is the nondimensional distance apart from the wall, $\kappa$ is the Von Karman constant that is equal to $0.4187, E$ is an empirical constant that is equal to $9.793, U_{\mathrm{p}}$ denotes the mean velocity, $k_{\mathrm{p}}$ represents the turbulence kinetic energy, $y_{\mathrm{p}}$ means the distance apart from the wall, $\mu_{\mathrm{m}}$ is the dynamic viscosity of the mixture fluid, and $y_{\lim }^{*}=11.225$ is the limiter to prevent from the deterioration of the numerical result.

The unsteady solver of "Ansys Fluent 19.2" is employed to resolve the equations and the finite volume method is adopted to discretize the computational domain. The time step of the unsteady calculation is set as $1 \mathrm{~ms}$. "SIMPLE" method is utilized to decouple and solve the velocity and pressure of the flow field. "Body force weighted" scheme is employed to discretize the pressure equation in this paper.

3.2. Computational Domain. Since the focus of this paper is to investigate the hydrodynamic characteristics of the BR and SR in the supercavitation flow and the supercavity deformations induced by the BR and SR, for the BR model, the attack angle of the HSSV is set as zero. The afterbodies of the HSSV including the fore-conic section, cylindrical section, and nozzle are completely enveloped in the supercavity and resultantly provide no effect on the hydrodynamic characteristics of the BRs and the supercavity shape. Thus, the afterbodies are excluded in the BR model for simplification, and only the BRs and cone cavitator are modeled. Different from the BR model, the SRs locate aft of the HSSV, and so the afterbodies must be included in the SR model. Additionally, two reference cases are also established by removing the BRs and SRs from the BR model and SR model to verify the effect of the $\mathrm{BR}$ and $\mathrm{SR}$ on the supercavity.

In the simulation of the supercavitation flow, the mass transfer process and the interface between the water phase and vapor phase are both sensitive to the disturbances of the pressure and velocity coming from the boundary of the flow field. Enlarging the dimension of the computational domain can decrease the impact and further increase the precision of the calculating results of the supercavity shape and hydrodynamic characteristics. To achieve the independent results, the diameter of the computational domain is at least 36 times larger than the maximum diameter of the supercavity [29]. The computational domain and boundary conditions are defined in Figure 4. In addition, the axial length of the computational domain also has an impact on the calculating results. The distances from the inlet to the cavitator and from the outlet to the closure point of the supercavity are assumed to be $L_{1}$ and $L_{2}$, respectively. The values of $L_{1}$ and $L_{2}$ will be confirmed in the section of "Validation."

As shown in Figure 4, an axial velocity of $100 \mathrm{~m} / \mathrm{s}$ is used as the inlet condition, a static pressure of $118,540 \mathrm{~Pa}$ is set as the outlet condition, and the operating depth is approximately equal to $1.76 \mathrm{~m}$. The cavitation pressure is set as $3540 \mathrm{~Pa}$, and the cavitation number is equal to 0.023 . The outside of the computational domain is regarded as a free slipping wall. The Froude number under the operation conditions is very high, so the effect of gravity force is not modeled in this research.

\subsection{Validation}

3.3.1. Validation of Axial Length of Computational Domain. The mass transfer between the liquid phase and the vapor phase is described by equations (7) and (8) and has direct correlation with the static pressure distribution of the flow field. Thus, the precise prediction of the static pressure is the basis and prerequisite for obtaining a reasonable supercavity shape. As shown in Figure 4, the velocity is set as $100 \mathrm{~m} / \mathrm{s}$ along the axial direction at the inlet, and the static pressure is changed to $118,540 \mathrm{~Pa}$ at the outlet. To weaken the impact of the boundary conditions on the calculating result, it is essential to keep a far enough distance from the inlet and outlet to the supercavity. However, extending the distance of inlet and outlet apart from the supercavity means the enlargement of the axial length of the computational domain and sequentially increases the computational cost. Thus, it is necessary to select a reasonable axial length for the computational domain. 


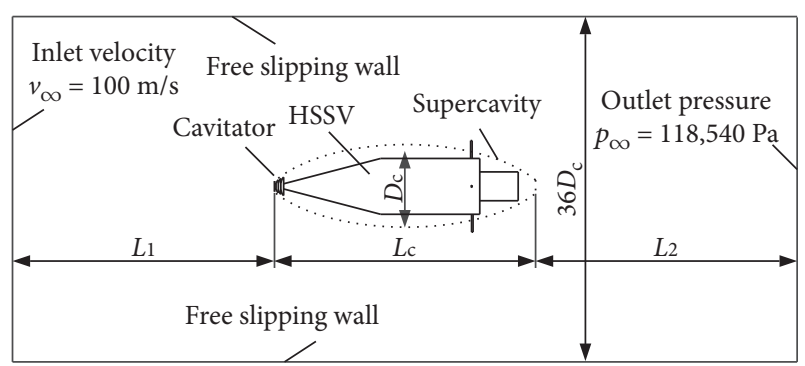

FIgURE 4: Computational domain and boundary conditions.

As shown in Figure 4, the distance from the inlet to cavitator is denoted as $L_{1}$ and that from the outlet to the closure point is $L_{2}$. Three different computational domains are designed by changing the length of $L_{1}$ and $L_{2}$ : $L_{1}=L_{2}=0.5 L_{\mathrm{c}}$ for the short model, $L_{1}=L_{2}=1.0 L_{\mathrm{c}}$ for the medium model, and $L_{1}=L_{2}=2.0 L_{\mathrm{c}}$ for the long model. Herein, $L_{\mathrm{c}}$ is approximately equal to the length of the supercavity and is estimated by the theoretical method. The corresponding numerical calculations are performed in the same condition as the BR model, and the supercavity profiles and static pressure at the axes of the three cases are compared in Figures 5 and 6, respectively.

It is illustrated by Figures 5 and 6 that no significant differences are found among the supercavities and static pressure distributions at the axes of the three different models when looking from an overall perspective. From the partial enlarged detail, some small differences can be discovered. The relative difference of the supercavity length between the short model and the medium model is about $1.1 \%$, and that between the medium model and long model is only $0.3 \%$. The maximum difference of the static pressure at the axis between the short model and medium model is about $5 \%$, and the static pressures at the axis of the medium model and long model are nearly the same. Therefore, it is regarded that the axial length of the medium model is long enough.

3.3.2. Grid Independence. The hexahedral mesh is utilized to build the topological structure of the computational domain. The grids near the walls are refined to ensure that $y$-plus locates in the range of $30-100$, which coincides with the requirement of the scale wall function and $k$-epsilon turbulence model. The operating condition of the rudder angle that is equal to zero is selected to perform the grid independence verification, and the coarse, medium, and refined meshes are, respectively, designed for the BR and SR models. By keeping the height of the first layer of grids near walls and the growth rate of the nodes on each edge unchanged, the nodes on each edge are increased to $\sqrt[3]{2}(\sim 1.260)$ times of the coarse mesh to achieve the medium mesh and increased to $\sqrt[3]{3}(\sim 1.442)$ to obtain the refined mesh. Thus, the node number of the medium mesh is about 2 times of the coarse mesh, and the node number of the refined mesh is approximately 3 times of the coarse mesh. For the BR model,

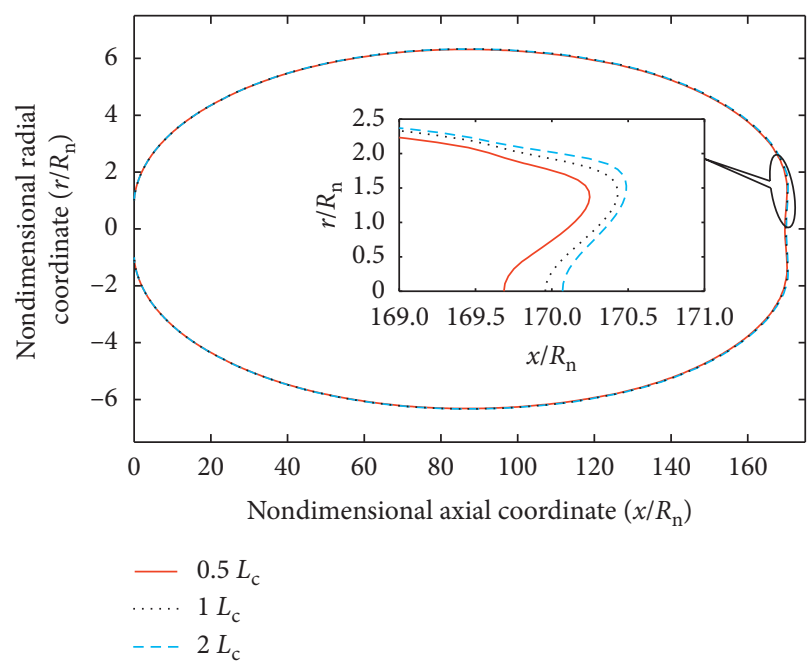

FIGURE 5: Comparison of supercavity profile.

the cell numbers of the coarse mesh, medium mesh, and fine mesh are 0.6 million, 1.2 million, and 1.8 million, individually. Then, the three meshes for the SR model have 0.8 million cells, 1.6 million cells, and 2.4 million cells, respectively.

In this research, attentions are mainly payed on the supercavity shape and the hydrodynamic characteristics of the rudder. Therefore, the grid is taken as independence when the calculated supercavity and hydrodynamic coefficient of the rudder change insignificantly with the increase of node number. The drag coefficients of the rudder and the supercavity lengths calculated with the different meshes of the BR model are compared in Figure 7, and the comparisons of the SR model are exhibited in Figure 8. Here, the drag coefficient is calculated by dividing the drag by the dynamic pressure and the projected area of the wetted part of the rudder. The supercavity length is normalized by the maximum radius of the HSSV.

As depicted in Figure 7, the relative difference of the drag coefficient between the fine and coarse meshes is $9.09 \%$, and it is $1.88 \%$ between the fine and medium meshes. The relative difference of the supercavity length of the BR model between the fine and coarse meshes is $7.11 \%$, and it is only $1.39 \%$ between the fine and medium meshes. For the SR model, by contrast with the drag coefficient of the rudder calculated by the fine mesh, the relative differences of the coarse mesh and medium mesh are $6.18 \%$ and $1.63 \%$, respectively. Moreover, compared to the supercavity length of the SR model simulated by the fine mesh, the relative differences of the coarse mesh and medium mesh are $4.94 \%$ and $1.12 \%$, respectively. The comparisons in Figures 7 and 8 demonstrate that the medium meshes of BR model and SR model satisfy the requirement of the grid independence. The individual medium mesh schemes are utilized to compute the hydrodynamic characteristics and supercavity shape of the BR model and SR model with different rudder angles. The medium meshes for the BR model and SR model are 


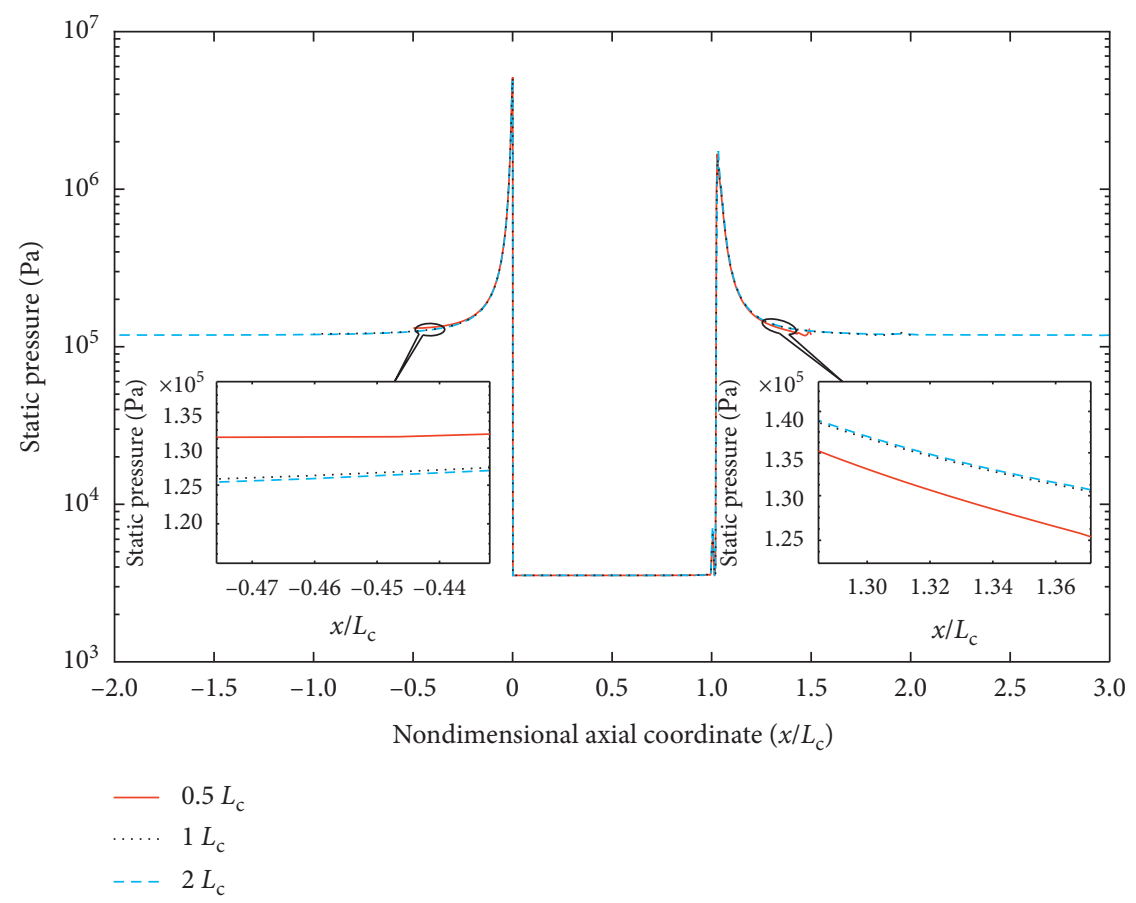

Figure 6: Comparison of static pressure at axis.

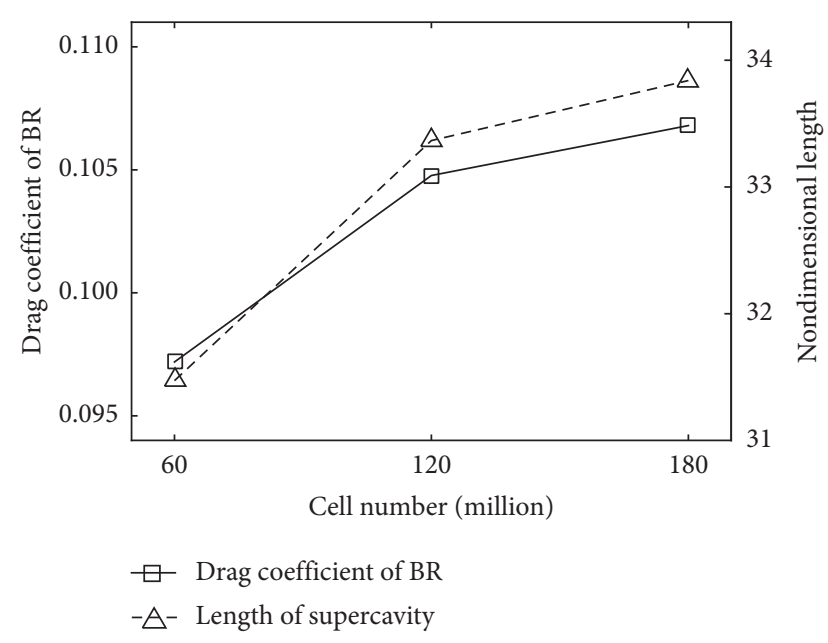

FIGURE 7: Grid independence verification of BR model.

depicted in Figures 9(a) and 9(b), respectively. Additionally, the grid independence verifications are performed for the reference models of the BR and SR model and are not covered here.

3.3.3. Validation of Supercavity. Savechenko carried out a series of sailing experiments to study the change law of supercavity along with the cavitation number in the range of $0.012-0.057$ and built the empirical formulas (13) and (14) to describe the relationship based on the experimental results [2]. According to the independent expansion principle presented by Logvinovich, the supercavity has the appearance of a quasiellipsoid, and the profile is described as

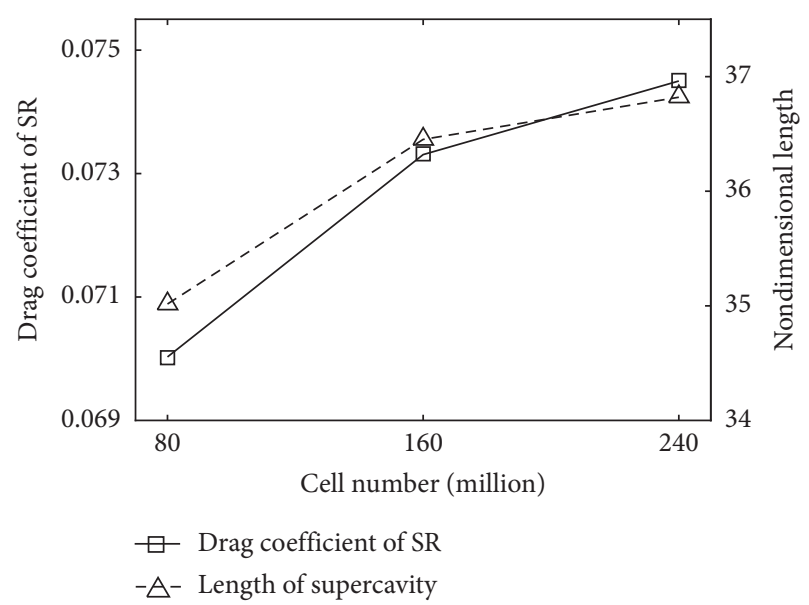

FIGURE 8: Grid independence verification of SR model.

formula (15). Equations (12)-(15) constitute a set of semiempirical correlations to predict the supercavity shape, which is well coincident with the experimental results. The semiempirical correlation has sufficiently experimental and theoretical basis and is widely used in the design of HSSVs $[14,20]$. Moreover, the cavitation number of the HSSV in this research is 0.023 exactly in the applicable range of the correlation. Therefore, equations (12)-(15) are regarded as experimental results to validate the numerical models.

$$
\begin{aligned}
\sigma & =\frac{p_{\infty}-p_{\mathrm{v}}}{0.5 \rho v^{2}}, \\
R_{\mathrm{c}} & =R_{\mathrm{n}} \sqrt{3.659+\frac{0.760}{\sigma}},
\end{aligned}
$$




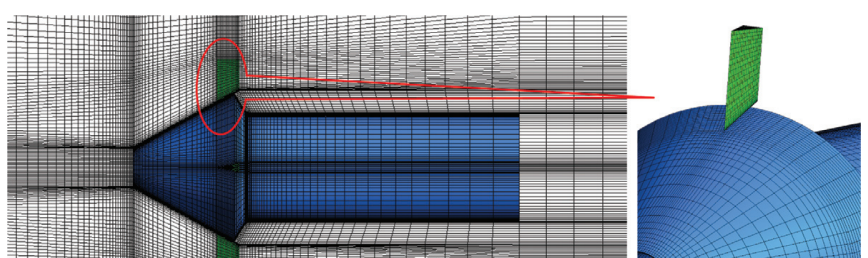

(a)

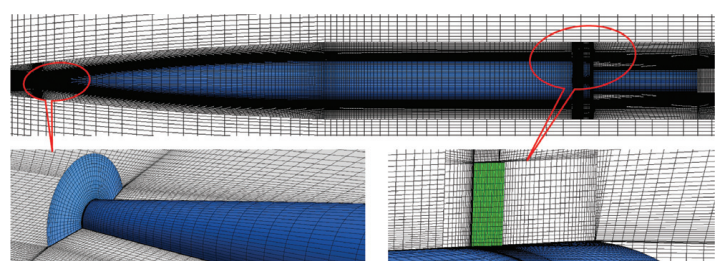

(b)

FIGURE 9: Computational mesh of BR model and SR model. (a) Mesh topology of BR model. (b) Mesh topology of SR model.

$$
\begin{aligned}
L_{\mathrm{c}} & =R_{\mathrm{n}}\left(4.0+\frac{3.589}{\sigma}\right), \\
R_{(x)} & =R_{\mathrm{c}} \sqrt{1-\left(1-\frac{R_{\mathrm{n}}^{2}}{R_{\mathrm{c}}^{2}}\right)\left(1-\frac{2 x}{L_{\mathrm{c}}}\right)^{2}},
\end{aligned}
$$

where $R_{\mathrm{n}}$ is the radius of the cavitators, $R_{\mathrm{c}}$ is the maximum radius of the supercavity, $L_{c}$ is the whole length of the supercavity, and $x$ represents the distance between the calculating section and cavitator.

To verify the accuracy of the numerical model, numerical simulations are performed for a $42 \mathrm{~mm}$ disk cavitator operating with the cavitation number in 0.017-0.029. The obtained supercavity profiles in different operating conditions are compared with the respective result of the empirical correlation, and the comparisons are shown in Figure 10. The axial and radial coordinates of the supercavity profiles are normalized by the radius of the cavitator $\left(R_{\mathrm{n}}=21 \mathrm{~mm}\right)$. Both of the axial and radial sizes of the numerical supercavity are slightly larger than those calculated by the empirical correlation. The nondimensional maximum radius and the full length of the supercavities are compared in Table 3. The maximum relative difference is less than $3 \%$. Good agreement is achieved. This confirms the suitability of the numerical model in predicting the supercavity shape.

3.3.4. Validation of Hydrodynamic Force. The experiments of a scaled HSSV model with SRs were performed in our previous work [24], and the hydrodynamic characteristics of $24^{\circ}$ wedge-shaped SRs in the supercavitation flow were achieved. The experiments were carried out in a water tunnel, and the supercavity was formed by artificial ventilation. In addition, the freestream speed is $10 \mathrm{~m} / \mathrm{s}$, operation pressure is $90 \mathrm{kPa}$, and the rudder angle changes from $0^{\circ}$ to $12^{\circ}$.

To validate the numerical model, the experiments are modeled and calculated. The numerical method proposed in this paper is used to compute the natural cavitation flow and cannot be directly applied to the simulation of the ventilation supercavitation flow. To calculate the flow field of the experiment, the numerical model is slightly modified by suppressing the cavitation model and adding an air inlet behind the cavitator. Then, all of the multiphase model, turbulence model, wall function, and model parameters remain unchanged. The experimental and numerical results of the hydrodynamic coefficients of the SR are compared in Figure 11. Regarding the lift coefficient and drag coefficient, the

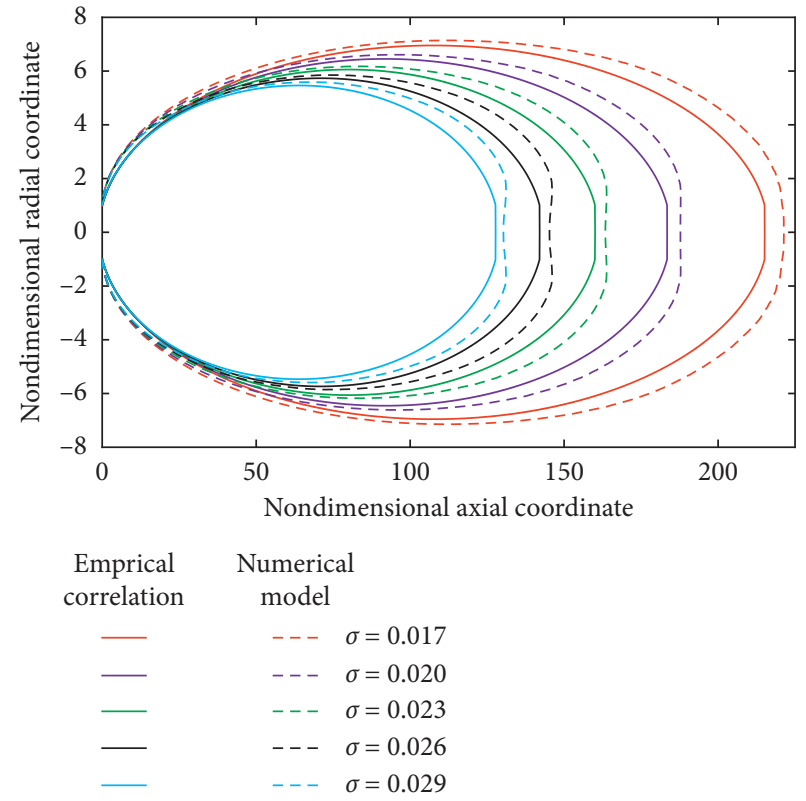

Figure 10: Comparison of supercavity profiles obtained by numerical model and empirical correlations. Solid line: empirical correlation; dashed line: numerical simulations.

numerical and experimental results have the same tendency. The lift coefficient suddenly decreases when the rudder angle is larger than $8^{\circ}$. Moreover, the maximum relative difference between the numerical and experimental lift coefficients is within $15 \%$, and that of the drag coefficient is no more than $10 \%$. Therefore, the numerical model can predict the hydrodynamic characteristics of wedge-shaped rudders.

\section{Results and Discussion}

4.1. BR Model. The vertical BR deflects from $0^{\circ}$ to $12^{\circ}$ with an interval of $1^{\circ}$, and the corresponding numerical calculations are carried out to achieve the hydrodynamic characteristics of the BR. The rudder angle is defined as positive when the rudder rotates around the pivot in clockwise direction and as negative if it turns reversely. Again, the supercavity deformations induced by the BR are achieved by comparison with the reference case.

4.1.1. Supercavity Deformation Induced by BR. The BR locates at the nose of the HSSV, disturbs the flow regime near the cavitator, and results in the supercavity deformations. 
TABle 3: Comparison of feature sizes of supercavity obtained by numerical model and empirical correlations.

\begin{tabular}{lcccccc}
\hline $\begin{array}{l}\text { Cavitation } \\
\text { number }\end{array}$ & $\begin{array}{c}\text { Numerical } \\
\text { model }\end{array}$ & $\begin{array}{c}\text { Full length } / R_{\mathrm{n}} \\
\text { Empirical } \\
\text { correlations }\end{array}$ & $\begin{array}{c}\text { Relative difference } \\
(\%)\end{array}$ & $\begin{array}{c}\text { Numerical } \\
\text { model }\end{array}$ & $\begin{array}{c}\text { Maximum radius } / R_{\mathrm{n}} \\
\text { Empirical } \\
\text { correlations }\end{array}$ & $\begin{array}{c}\text { Relative difference } \\
(\%)\end{array}$ \\
\hline 0.017 & 221.33 & 215.12 & 2.89 & 7.14 & 6.95 & 2.73 \\
0.020 & 187.76 & 183.45 & 2.35 & 6.61 & 6.45 & 2.48 \\
0.023 & 163.38 & 160.04 & 2.09 & 5.85 & 5.73 & 2.98 \\
0.026 & 145.24 & 142.03 & 2.26 & 5.59 & 5.46 & 2.09 \\
0.029 & 130.29 & 127.75 & 1.99 & & & 2.38 \\
\hline
\end{tabular}

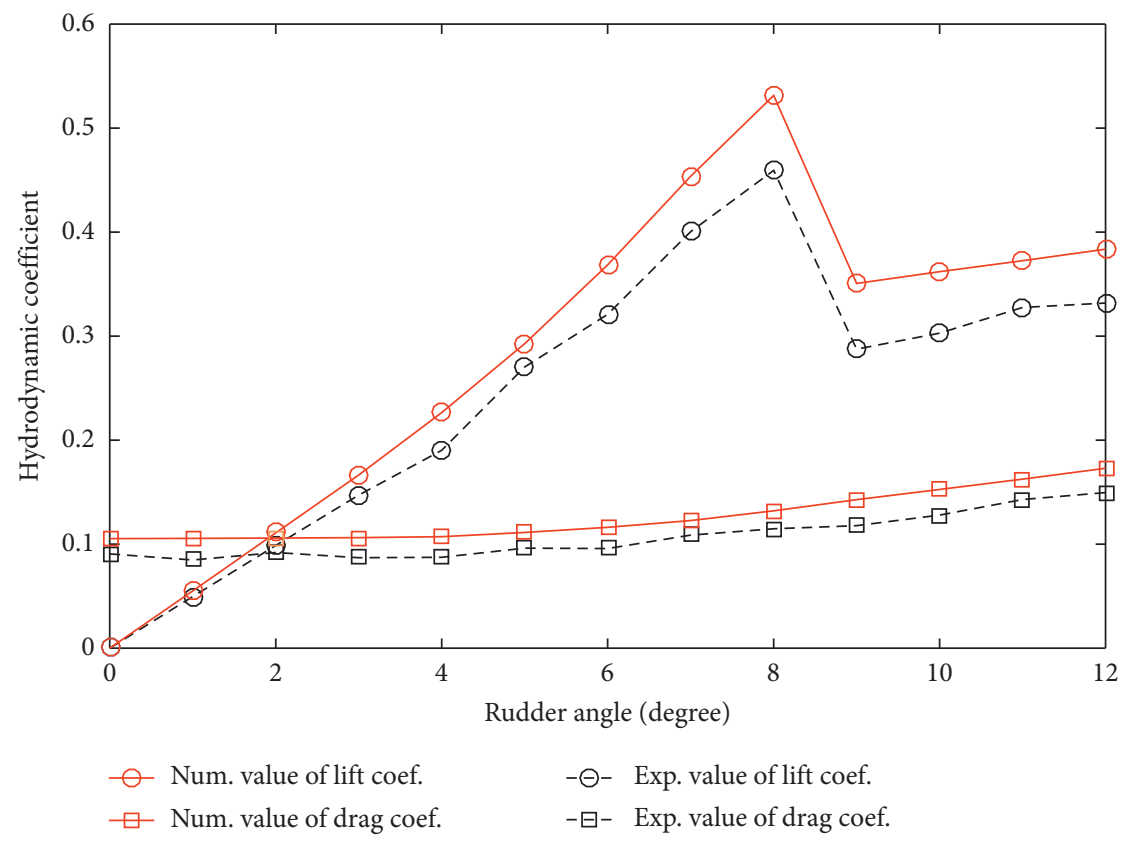

FIGURE 11: Comparison of experimental and numerical hydrodynamic coefficients of SR.

For a practical HSSV, the supercavity shape is closely related to the planing force acting on the hull and expected to be predictable [14]. Therefore, it is imperative to evaluate the influence of the BR on the supercavity. According to the numerical results, the isosurface of the vapor volume fraction equal to 0.5 is regarded as the supercavity boundary. To identify the influence of the BR on the supercavity, the three-dimensional views of the supercavities produced by the BR model and the reference model are compared in Figure 12.

The supercavity produced by the cone cavitator with the BRs still maintains the appearance of a quasiellipsoid, but the BRs lead to an obvious deformation to the forepart of the supercavity. A zoom-in view near the cavitator is shown in Figure 12(c). It is indicated that each BR produces a small cavity behind itself, and the small cavity turns into a concave deformation on the supercavity. The BR with sharp edges can be regarded as a small cavitator, so a small cavity is formed behind it. For the reason that the cavity behind the BR is far smaller than the supercavity, it is closed quickly and merges into the supercavity. Moreover, a high-pressure region is produced behind the closure point, which results in the concave deformation on the supercavity. The high pressure weakens gradually with moving away from the closure point as well as the concave deformation. Then, the concave deformation finally disappears near the middle section of the supercavity. Kuklinski and Fredette [23] experimentally investigated the supercavities generated by snowflake-shaped and star-shaped cavitators in a water tunnel, and the convex and concave deformations of the supercavity were observed exactly behind the bulges of the cavitator. Similar phenomenon is also found in Figure 12(c).

It is shown in Figure 12 that the most significant deformation of the supercavity locates exactly behind the BR. The deformation gradually weakens as it is far away from the cavitator. Moreover, the supercavity profiles at different longitudinal sections and cross sections are compared in Figures 13 and 14, respectively. Also, the supercavity profiles are normalized by the radius of the HSSV $\left(R_{\mathrm{c}}\right)$. The supercavity profile of the reference case is also plotted in Figure 13. Since the cavitator of the reference case is a revolved body, the supercavity profiles at different longitudinal sections are identical. As shown in Figure 13, section I corresponds to the longitudinal section locating in the vertical plane, where section I is a longitudinal section with an intersection angle of $45^{\circ}$ relative to section II. 


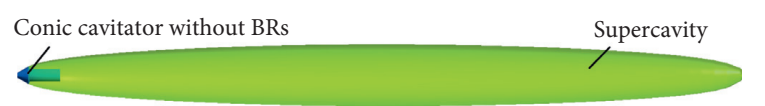

(a)

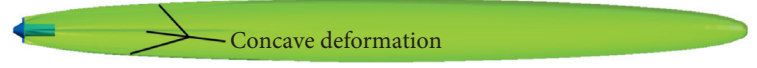

(b)

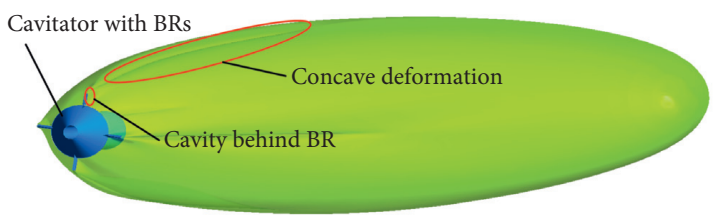

(c)

FIGURE 12: Supercavities produced by cone cavitator with or without BRs. (a) Supercavity produced by cavitator without BRs (left view). (b) Supercavity produced by cavitator with BRs (left view). (c) Supercavity produced by cavitator with BRs (left-front view).

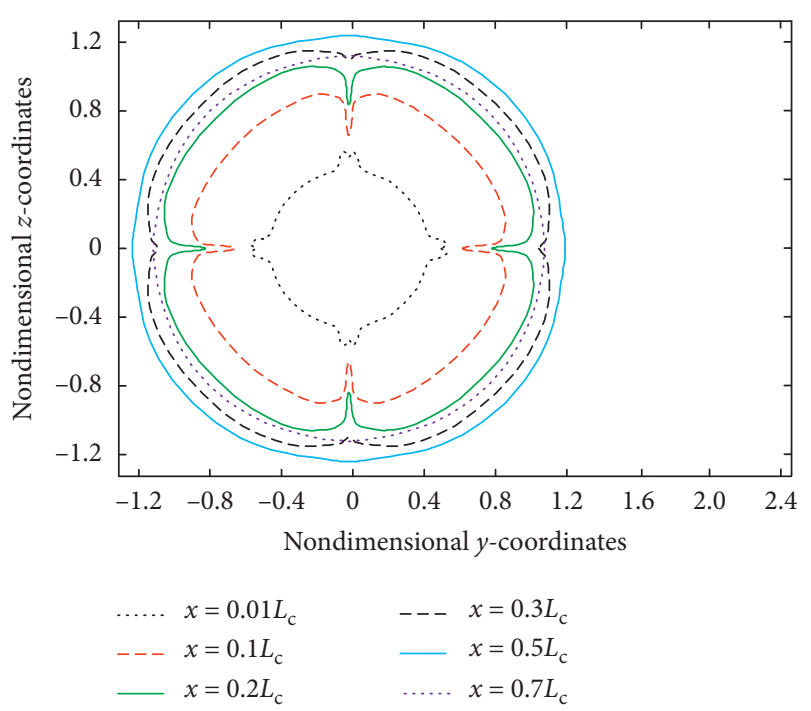

FIGURE 13: Supercavity profiles on different longitudinal sections.

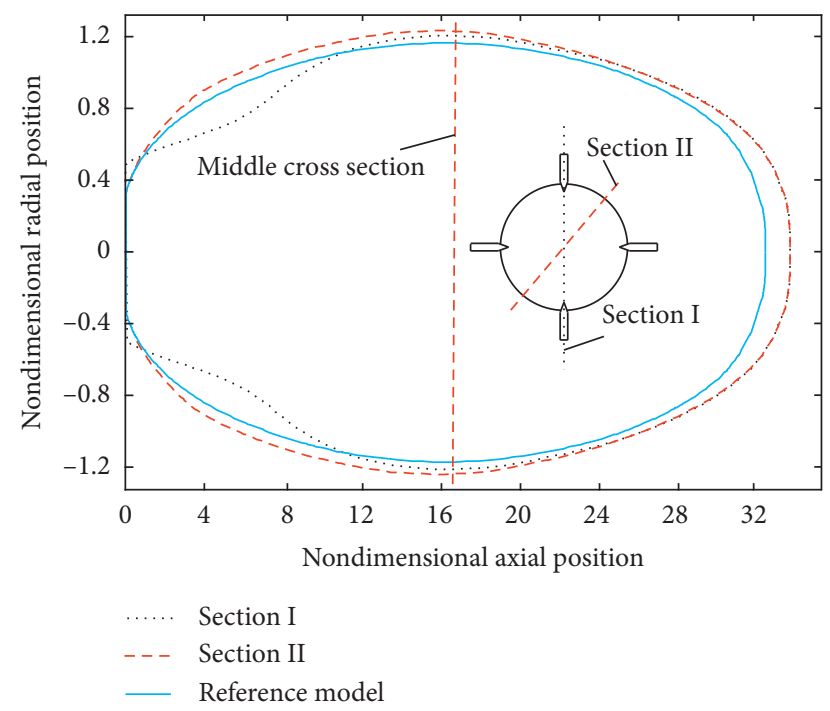

FIGURE 14: Supercavity profiles of different cross sections.

It is shown in Figure 14 that both of the supercavity profiles at section I and section II are larger than those of the reference case except for the forepart. The relative differences of the full length and maximum radius are less than $4.3 \%$ and $3.4 \%$, respectively. For section I, the supercavity shape is significantly changed by the BRs and shows a nonsymmetric shape about the middle cross section. For section II, the supercavity profile still maintains a quasiellipse and is slightly affected by the BR. The deformations mainly concentrate upon the forepart of the supercavity, especially the regions exactly facing the BR. Moreover, the difference between the supercavity profiles of section I and section II disappears behind the maximum cross section.

Figure 14 depicts the supercavity profiles at six different cross sections, namely, $0.01 L_{\mathfrak{c}}, 0.1 L_{\mathfrak{c}}, 0.2 L_{\mathfrak{c}}, 0.3 L_{\mathfrak{c}}, 0.5 L_{\mathfrak{c}}$, and $0.7 L_{\mathrm{c}}$. Herein, $L_{\mathrm{c}}$ is the supercavity length equal to $33.2 R_{\mathrm{c}}$. It is found that the BR changes the intrinsic circular cross section of the supercavity, especially for the forepart. The convex deformations $\left(0.01 L_{\mathrm{c}}\right)$ and concave deformations $\left(0.1 L_{\mathfrak{c}}, 0.2 L_{\mathcal{c}}\right.$, and $\left.0.3 L_{\mathrm{c}}\right)$ on the supercavity exactly behind the BR are observed. The BR induces a small cavity at the origin of the supercavity. Subsequently, the small cavity is closed and turns into a concave deformation at the section at $0.1 L_{c}, 0.2 L_{c}$, and $0.3 L_{c}$. Then, at the middle cross section $\left(0.5 L_{\mathcal{c}}\right.$, as shown in Figure 13), the influence of the BR on the supercavity is negligible. In addition, the supercavity profile at the cross section is slightly asymmetrical about the middle cross section. The supercavity profile at the section of $0.3 L_{c}$ is slightly larger than that of $0.7 L_{\mathrm{c}}$.

From the above discussions, though the BR affects the supercavity shape, the deformations mainly exist at the forepart and turn to be very limited behind the middle section.

4.1.2. Hydrodynamic Characteristics of BR. The hydrodynamic force acting on the rudder is determined by the wetted area and rudder angle. Then, the force can be decomposed into the drag and lift components. The lift is always regarded as a control force to regulate the motion of HSSVs. Since the BR locates before the origin of the supercavity, it is fully wetted, and its wetted area remains unchanged. The drag forces and lift forces generated by the BR at different rudder angles can be obtained from the numerical results. Then, these forces are normalized by equation (16). The lift coefficient, drag coefficient, and lift-to-drag ratio of the BR are shown in Figure 15. 


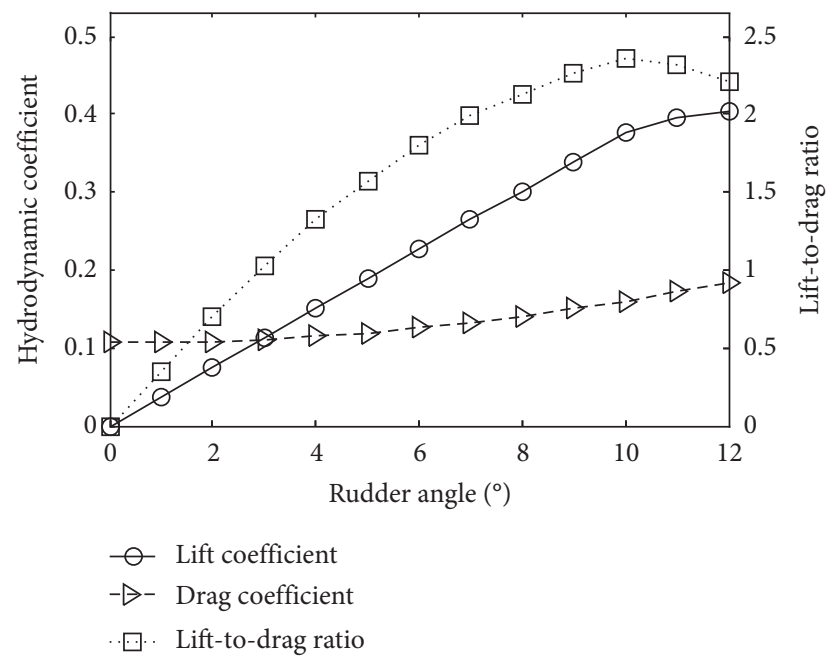

Figure 15: Hydrodynamic characteristics of BR.

$$
C_{F_{\mathrm{R}}}=\frac{F_{\mathrm{R}}}{(1 / 2) \rho b \bar{h} v_{\infty}^{2}},
$$

where $F_{\mathrm{R}}$ is the lift force or drag force acting on the rudder, $C_{F_{\mathrm{R}}}$ denotes the drag or lift coefficient of the rudder, $\rho$ is the water density, $v_{\underline{Q}}$ is the freestream speed, $b$ is the chord of the rudder, and $\bar{h}$ is the average wetted length of the rudder, which can be obtained by dividing the projected area of the wetted part by the chord length.

The lift coefficient of the BR shows an approximate linearity with the increase of the rudder angle. It is worth noting that the slope slightly decreases when the rudder angle is greater than $10^{\circ}$. The drag coefficient of the BR remains unchanged from $0^{\circ}$ to $5^{\circ}$, but the magnitude increases by approximately $50 \%$ from $5^{\circ}$ to $12^{\circ}$. In addition, the lift-to-drag ratio is not monotonous with the increase of the rudder angle. A peak ratio of 2.4 is obtained when the rudder angle equals $10^{\circ}$.

To explain the reason resulting in the nonlinear lift coefficient as exhibited in Figure 15, the vapor phase distributions on the suction surface of the BR with rudder angles of $10^{\circ}, 11^{\circ}$, and $12^{\circ}$ are extracted and compared in Figure 16 . When the rudder angle exceeds $10^{\circ}$, the minimum pressure at the suction surface drops to the cavitation pressure, and the partial cavitation occurs. Moreover, the area covered by the cavity further increases with the increase of the rudder angle. To provide more details, the middle section is chosen as shown in Figure 16(b) to display the static pressure distribution on the $\mathrm{BR}$. The static pressure is normalized to be the pressure coefficient and plotted in Figure 17. Generally, with the increase of the rudder angle, the pressure on the pressure surface increases, and the pressure on the suction surface drops. However, if the cavitation occurs at the suction surface, the minimum static pressure is equal to the cavitation pressure and cannot drop continuously. Moreover, if the suction surface is fully covered by the cavity, the static pressure remains unchanged. Considering that the lift force of the BR derives from the pressure difference between the pressure surface and the suction surface, the growth rate of the lift coefficient of course drops gradually when the rudder angle exceeds $10^{\circ}$.

4.2. SR Model. Similarly, the hydrodynamic characteristics of the SR are obtained under the conditions of the rudder angle within $0^{\circ}-12^{\circ}$. Also, the influence of the SR on the supercavity shape is confirmed by comparison with the corresponding reference model. Herein, the definition of the sign rudder angle is the same as that of the BR model.

4.2.1. Influence of SR on Supercavity. The HSSV without SRs is used as a reference to study the influence of the SR on the supercavity shape. The supercavities of the HSSVs with and without the SRs are depicted in Figures 18(a) and 18(b). Even though the partial supercavity after the SR is disturbed as shown in Figures 18(a) and 18(b), the nozzle is still entirely enveloped in the supercavity. The corresponding longitudinal profiles are normalized by the radius of HSSV and are shown in Figure 19. As shown in Figure 19, the supercavity of the SR model is approximately the same as that of the reference model in the region before the SR and totally different in the region behind the SR. In addition, though the tail of the supercavity deforms significantly, all the hulls of the HSSV including the nozzle are still fully covered by the supercavity.

To provide more details, four cross sections (sections I-IV) near the SR are chosen to analyze the supercavity deformations induced by the SR. The positions of the four cross sections are also shown in Figure 19. Section I and section II are $2.13 R_{\mathrm{c}}$ and $0.10 R_{\mathrm{c}}$ ahead of the leading edge of the SR, respectively. Then, section III and section IV are $0.80 R_{\mathrm{c}}$ and $3.17 R_{\mathrm{c}}$ behind the leading edge of SR and correspond to the end of the cylindrical section and the middle position of the nozzle, respectively. The cross section profiles of the supercavities produced by the HSSV with and without SRs are compared in Figure 20. Herein, the comparisons at section I and section II are shown in Figure 20(a), and the 


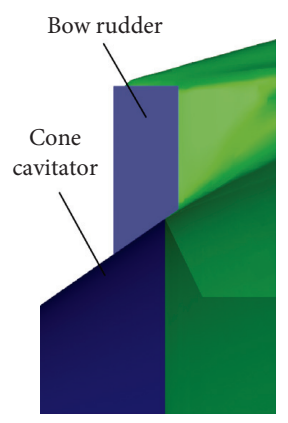

(a)

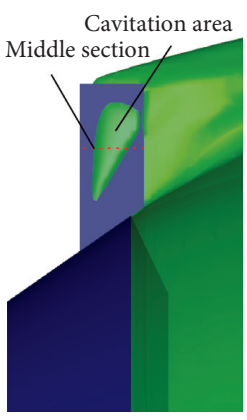

(b)

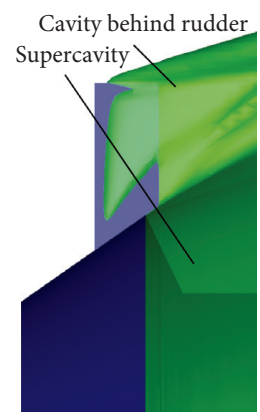

(c)

Figure 16: Comparison of cavity on suction surface of BR in different rudder angles. (a) Rudder angle: $10^{\circ}$. (b) Rudder angle: $11^{\circ}$. (c) Rudder angle: $12^{\circ}$.

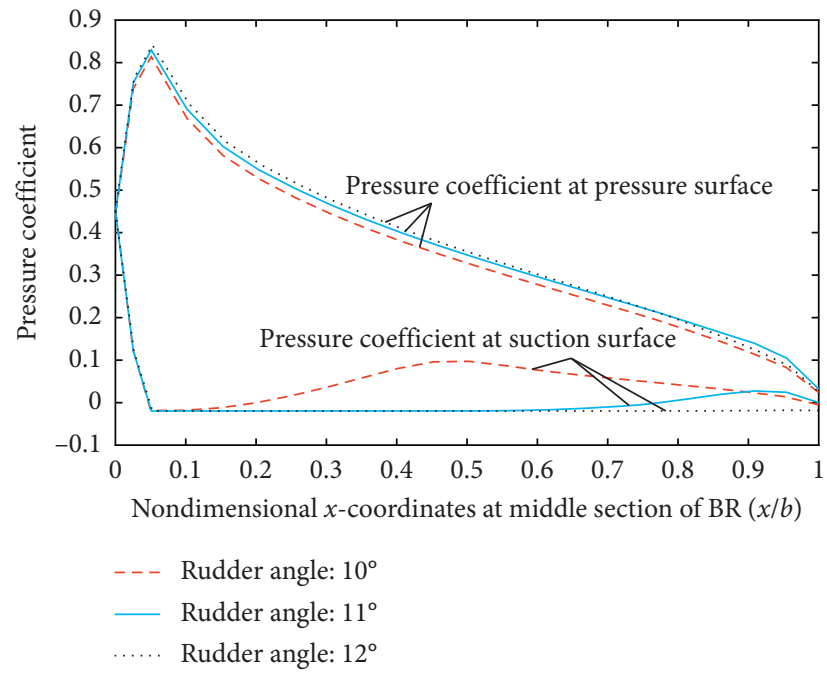

FIGURE 17: Comparison of static pressure distribution on BR in different rudder angles.

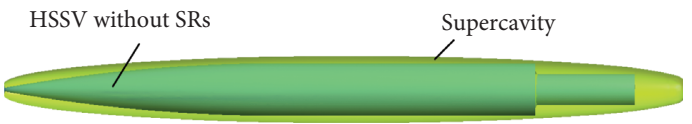

(a)

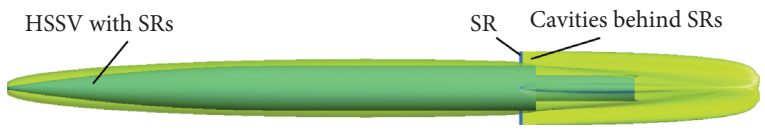

(b)

FIGURE 18: Overall supercavities generated by HSSV with and without SRs. (a) Supercavity produced by HSSV without SRs. (b) Supercavity produced by HSSV with SRs.

comparisons at section III and section IV are shown in Figures 20(b) and 20(c), respectively.

As shown in Figure 20(a), the supercavity profiles of the SR model and the reference model at section I are almost the same, and both of the two supercavity profiles remain round. Moreover, the same phenomenon can be found as well for section II. It is shown in Figures 20(b) and 20(c) that the cross-section profiles of the supercavity at section III and section IV are changed by the SRs, and the difference between the SR model and the reference model is obvious. For the SR model, each SR produces a small cavity behind itself; these small cavities expand and merge into the supercavity gradually. The development of the supercavity and the propagation of the disturbance in the flow field are both along the streamwise, so the supercavity shape and flow regime before the SRs are hardly influenced and behind the SRs are obviously changed. For a practical HSSV, the flow regime in the region near the closure point of supercavity is mainly influenced by the gas-leakage and engine jet, so the supercavity shape at the region near and behind the nozzle outlet is not covered in this research.

Although the supercavity before the SR is nearly not affected, an obvious concave deformation of supercavity is found around the SR as shown in Figures 21(a) and 21(b). Reference [24] experimentally investigates the supercavitation flow regime around an HSSV model with the SR 

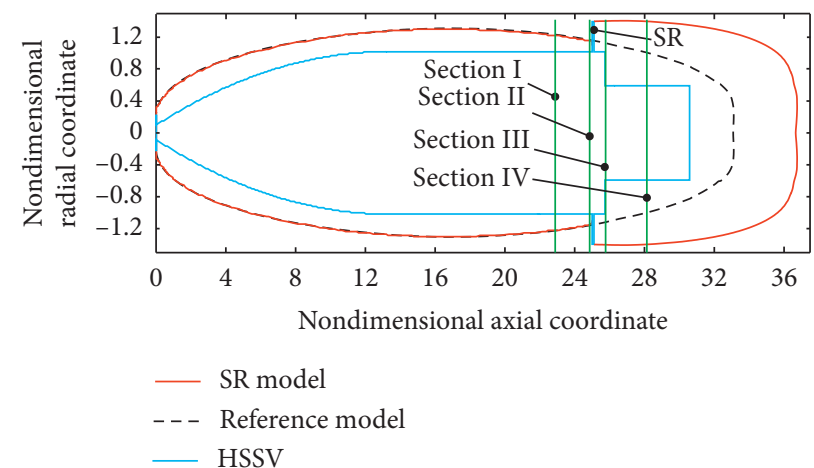

FIgURE 19: Comparison of supercavity profiles of HSSV with and without SRs.
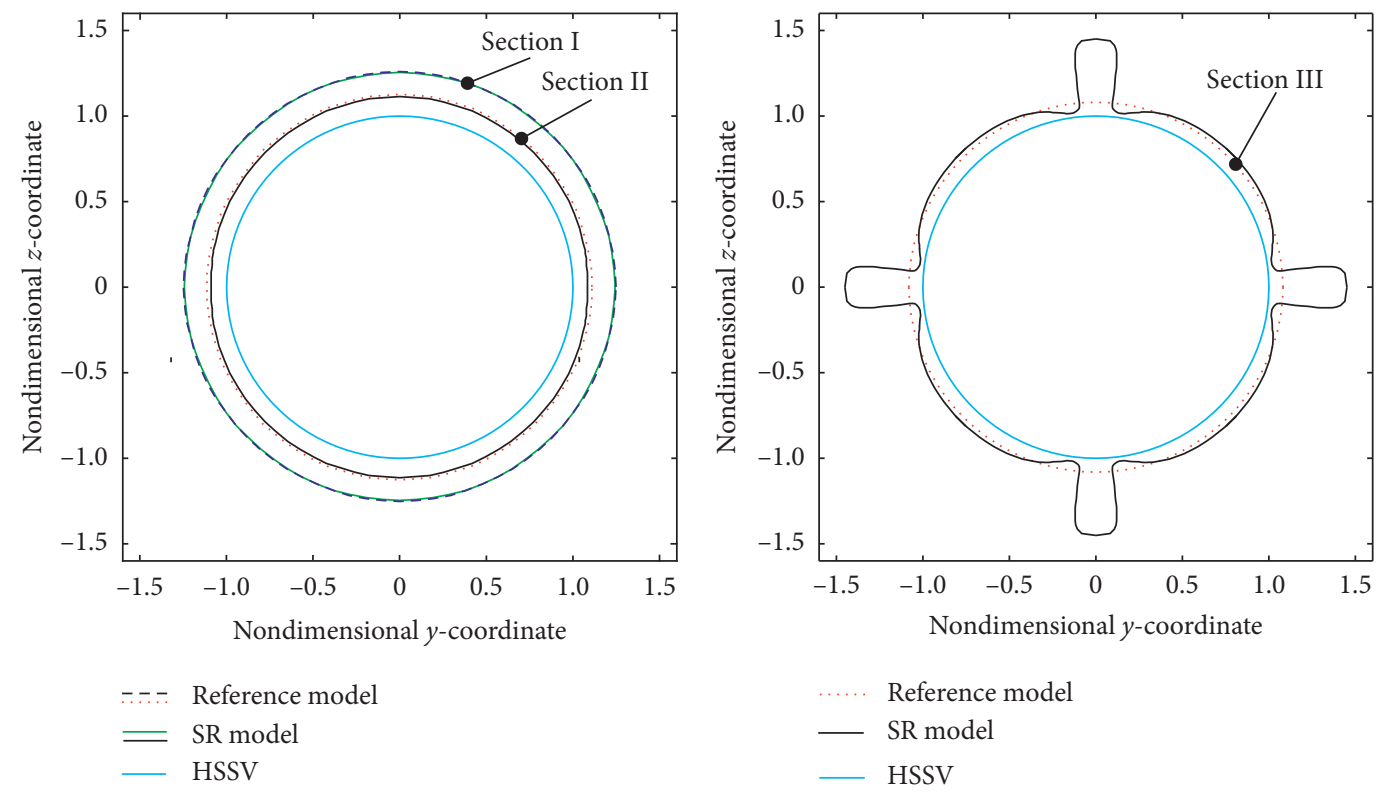

(a)

(b)

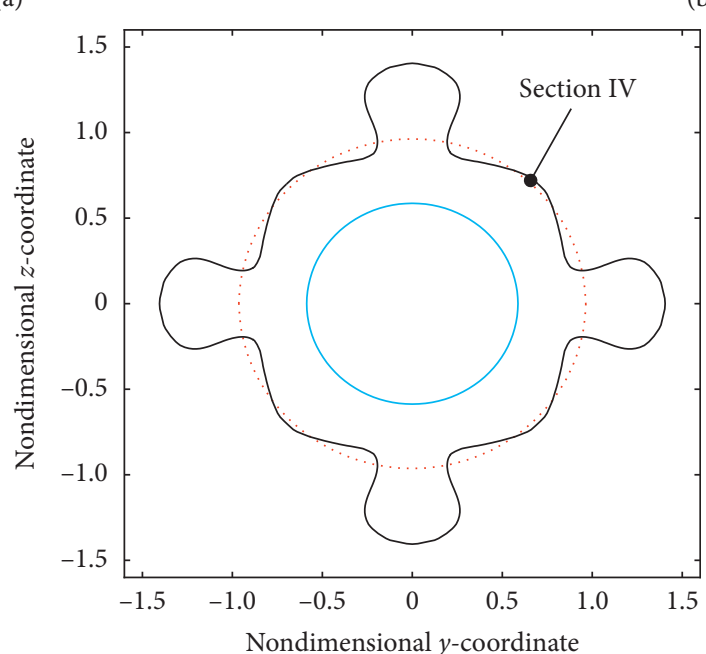

Nondimensional $y$-coordinate

Reference model

- SR model

- HSSV

(c)

FIgURE 20: Comparison of cross-section profiles of supercavities produced by HSSV with and without SRs. (a) Comparisons at sections I and II. (b) Comparisons at section III. (c) Comparisons at section IV. 


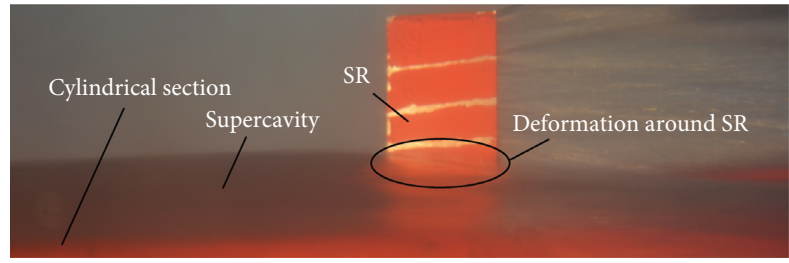

(a)

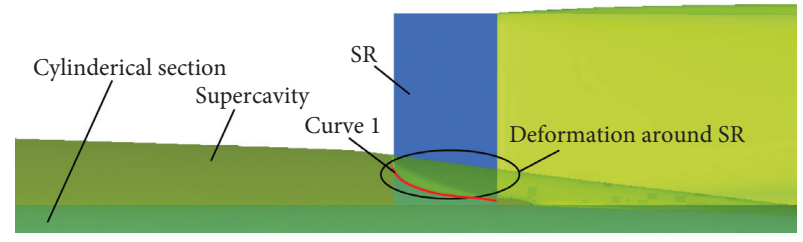

(b)

FIGURE 21: Experimental and numerical results about concave deformation of supercavity near SR. (a) Experimental result of supercavity deformation around SR. (b) Numerical result of supercavity deformation around SR.

in the water tunnel and obtains the supercavity shape near the SR as shown in Figure 21(a). The numerical results of the SR model also demonstrate the partial supercavity deformation around the SR and are exhibited in Figure 21(b).

As shown in Figure 21, both of the experimental and numerical results demonstrate that the SR can induce the concave deformation on the supercavity near the SR. Moreover, curve 1 plotted in Figure 21(b) is the intersecting line between the deformed supercavity and the SR. The intersecting line is obviously below the top profile of the supercavity, so the wetted area of the SR increases greatly by considering the actual supercavity deformation. To quantify the increment of the wetted area of the SR, the practical supercavity profile near the SR is plotted in Figure 22, and the undisturbed supercavity profile is also displayed as a reference. The axial and radial sizes of supercavity profile are normalized by the radius of the HSSV. It is shown that the deformed supercavity leads to the obvious increase of the wetted area of the SR. As shown in Figure 22, the red-dashed line denotes the undisturbed supercavity profile, so the area of the rudder above the dashed line is the ideal wetted area of the SR. Similarly, the black solid line represents the deformed supercavity profile near the SR, and the area of the SR above the solid line is the practically wetted area of the SR. Moreover, taking the $17.5 \mathrm{~mm} \times 46 \mathrm{~mm}$ SR listed in Table 1 as an example, the ideal wetted area is $549.8 \mathrm{~mm}^{2}$, and the actual wetted area is $711.4 \mathrm{~mm}^{2}$. When the supercavity deformation is considered, the wetted area of the SR increases by $29.4 \%$. This concludes that the supercavity deformation near the SR must be taken into account.

4.2.2. Hydrodynamic Characteristics of SR. The SR of the HSSVs is wetted by puncturing the supercavity, and the control force is produced at the wetted part. According to the numerical results of the SR model, the hydrodynamic forces acting at the SR with the rudder angle of $0^{\circ}-12^{\circ}$ are achieved and normalized by using equation (16). The nondimensional lift and drag coefficients are shown in Figure 23. The lift force consists of two linear piecewise sections. The slope of the lift coefficient, also named a rudder efficiency, in $0^{\circ}-6^{\circ}$ is approximately four times larger than that in the range of $7^{\circ}-12^{\circ}$. The lift coefficient of the SR of $6^{\circ}$ is 0.18 and suddenly drops to 0.15 when the rudder angle increases to $7^{\circ}$. Moreover, all the lift coefficients in $7^{\circ}-12^{\circ}$ are all less than that of $6^{\circ}$. In addition, the sudden drop of the lift coefficient of wedge-shaped rudders is also verified in the

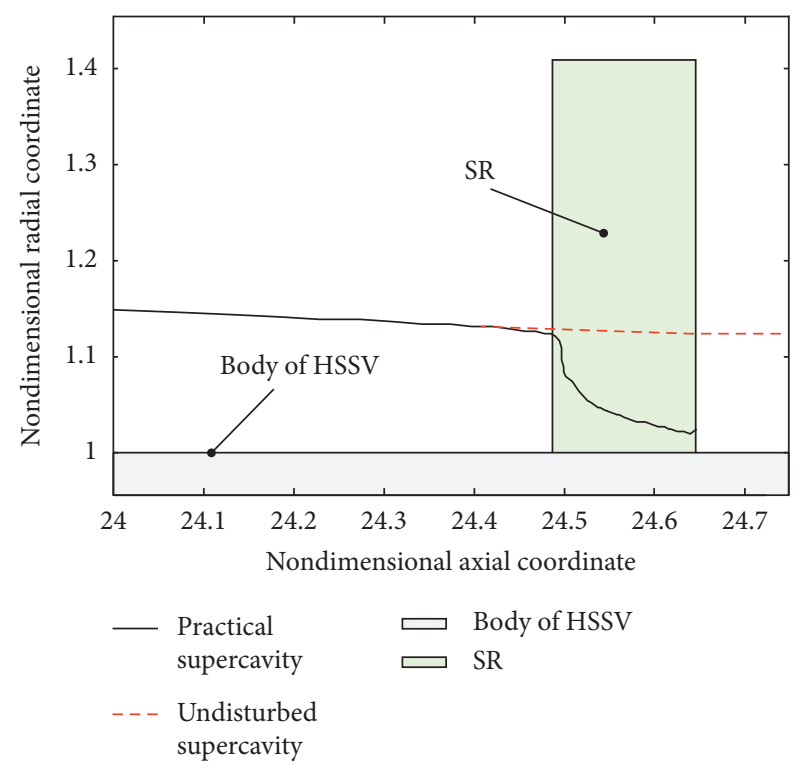

FIGURE 22: Actual wetted condition of SR.

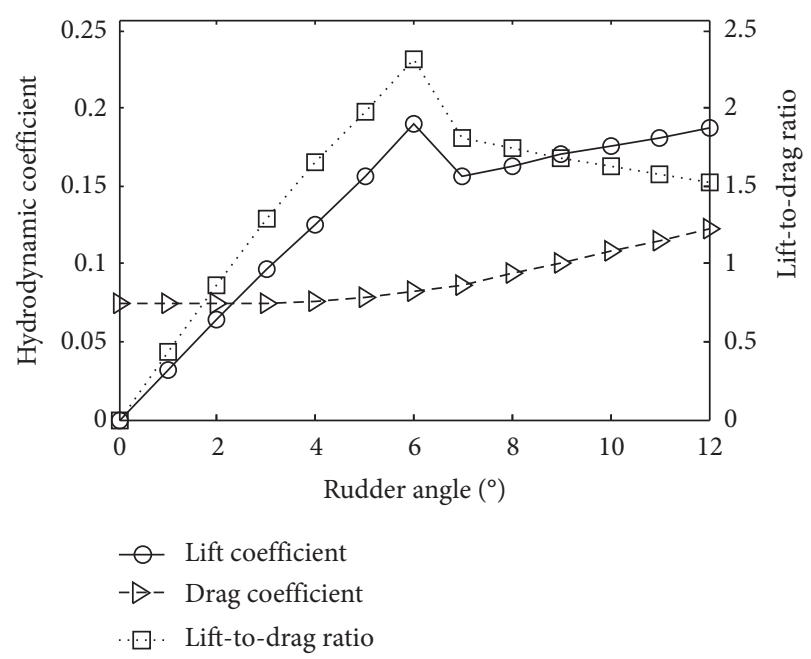

FIgURE 23: Hydrodynamic characteristics of SR.

ventilated supercavitation experiments performed in water tunnels $[24,25]$. The drag coefficient changes slightly as the rudder angle increases from $0^{\circ}$ to $5^{\circ}$ and increases by about $50 \%$ from $5^{\circ}$ to $12^{\circ}$. The drag characteristic is similar to that of the BR. The lift-to-drag ratio reaches the peak value when 
the rudder angle equals $6^{\circ}$. Figure 23 depicts that the ability of the SR generating the lift force weakens suddenly when the rudder angle exceeds $6^{\circ}$. This implies that the rudder angle of the SR is better no more than $6^{\circ}$ for the condition of this research, and this value can be regarded as the critical rudder angle.

To explain the reasons causing the sharp decrease of the lift coefficient, the distributions of the static pressure and vapor phase at the middle of the SR are extracted and compared. The volume fraction contours of the vapor phase near the SR when the rudder angle equals $6^{\circ}$ and $7^{\circ}$ are shown in Figure 24(a), and the static pressure contours are compared in Figure 24(b). Similar to the lift coefficient, both of the vapor phase distribution and static pressure contours change suddenly when the rudder angle increases from $6^{\circ}$ to $7^{\circ}$. No vapor phase is found at the suction and pressure surface of the SR for the rudder angle equal to $6^{\circ}$, and the static pressure decreases along the streamwise at the suction surface and increases at the pressure surface. However, when the rudder angle increases to $7^{\circ}$, the suction surface is suddenly completely covered by cavity, and the flow regime changes obviously. Then, the static pressure at the suction surface is equal to the cavitation pressure. Moreover, by contrast with the condition of rudder angle equal to $6^{\circ}$, the maximum pressure at the pressure surface is slightly increased and the minimum pressure is obviously decreased when the rudder angle is set to be $7^{\circ}$. Resultantly, the average pressure at the pressure surface decreases when the rudder angle exceeds the critical value. Moreover, the decrease of the static pressure at the suction surface is limited because of the full cavitation. As a result, the lift produced by the SR drops suddenly as the rudder angle becomes greater than the critical value.

As mentioned in Section 4.1.2, the lift force derives the pressure difference between the suction pressure and the pressure surface. The complete cavitation prevents the continuous decrease of the static pressure at the suction surface with the increase of the rudder angle. Therefore, the growth rate of the lift coefficient also decreases when the rudder angle is larger than the critical value.

4.3. Performance Comparison between BR and SR. The influences of the BR and SR on the supercavity shape as well as their hydrodynamic characteristics are discussed in Sections 4.1 and 4.2. Then, the merits and demerits of the BR and SR are summarized and compared to propose a suggestion to the arrangement of the control surface of HSSVs.

The drag force acting on the cavitator of the BR model is slightly larger than that of the reference model because of the four BRs. Thus, the BR model generates a slightly larger supercavity when compared with the reference model. The BRs also lead to a noncircular cross section of the forepart of the supercavity and induce a slight asymmetry to the supercavity about the middle section. The deformation of the supercavity mainly concentrates on the forepart and is very limited behind the middle section. By contrast, the SR exerts a tiny influence on the size and shape of the supercavity before it, but the partial concave deformation on the supercavity around the SR is observed. Moreover, the concave deformation increases the wetted area of the SR by $29.4 \%$. In addition, the rudder efficiency in the supercavitation flow is largely determined by the wetted area. BRs are almost fully wetted, and the lift force is only related to the rudder angle. Unlike the $\mathrm{BR}$, the $\mathrm{SR}$ is partially wetted, except for the rudder angle; the lift force also depends on the actual wetted area. Thus, the concave deformation of the supercavity around the SR must be taken into account because it largely increases the wetted area of the SR.

The lift coefficient of the BR increases with the rudder angle from $0^{\circ}$ to $12^{\circ}$ monotonously. However, the lift coefficient of the SR increases linearly along with the rudder angle in $0^{\circ}-6^{\circ}$ and $7^{\circ}-12^{\circ}$. The peak lift coefficient of the SR is obtained at the rudder angle of $6^{\circ}$. Assuming that the rudder angles of the SR and BR are both in the range of $0^{\circ}-12^{\circ}$, the maximum lift coefficient of the BR is 0.4 , and that of the SR is 0.18 . The corresponding rudder angle for the $\mathrm{BR}$ is $12^{\circ}$, and that for the SR is $6^{\circ}$. Then, the corresponding drag coefficient of the former is approximately 2.1 times larger than the latter. Considering the geometrical features of the HSSV listed in Table 1, the moment arm of the BR is about 2 times longer than that of the SR. Therefore, the maximum control moment produced by the unit wetted area of the BR is approximately 4.4 times larger than that of the SR. Then, it concludes that the operation efficiency of the BR is higher than the SR.

The partial pressure near the front surface of the cone cavitator is far higher than the ambient pressure due to the stagnation of the coming flow. This results in the BR locating in a place where the static pressure is much higher than the far field pressure. Therefore, the cavitation does not occur at the suction surface of the BR until the rudder angle exceeds $10^{\circ}$. Moreover, the partial cavitation occurs and gradually enlarges at the suction surface of the BR, which results in the decrease of the growth rate of the lift coefficient. By contrast with the BR, the SR is mounted near the tail of the supercavity; the static pressure near the SR is lower than the freestream. Then, the static pressure at the suction surface of the SR further decreases with the increase of the rudder angle, and the cavitation suddenly occurs at all the suction surface when the rudder angle exceeds $6^{\circ}$. Unlike the BR, the suction surface of the SR is fully covered by cavity, while the rudder is larger than the critical value, and the pressure contours at the pressure surface are also largely changed. As a result, the lift coefficient suddenly drops by $16.7 \%$ and the growth rate nearly decreases by $75 \%$.

As mentioned above, the SR is partially wetted, and some challenges still exist in the HSSVs using the SR layout scheme. Except for the rudder angle, the lift coefficient of the $\mathrm{SR}$ is also largely influenced by its actual wetted area. The actual wetted area of the SR is directly determined by the position, size, and shape of the supercavity at the corresponding position, and the calculation procedure is very complicated. For an HSSV moving unsteadily, the position and size of the supercavity can be calculated according to the previous motion status of the HSSV by applying the theoretical method [30]. The supercavity deformation near the SR and the influence on the wetted area are provided in 

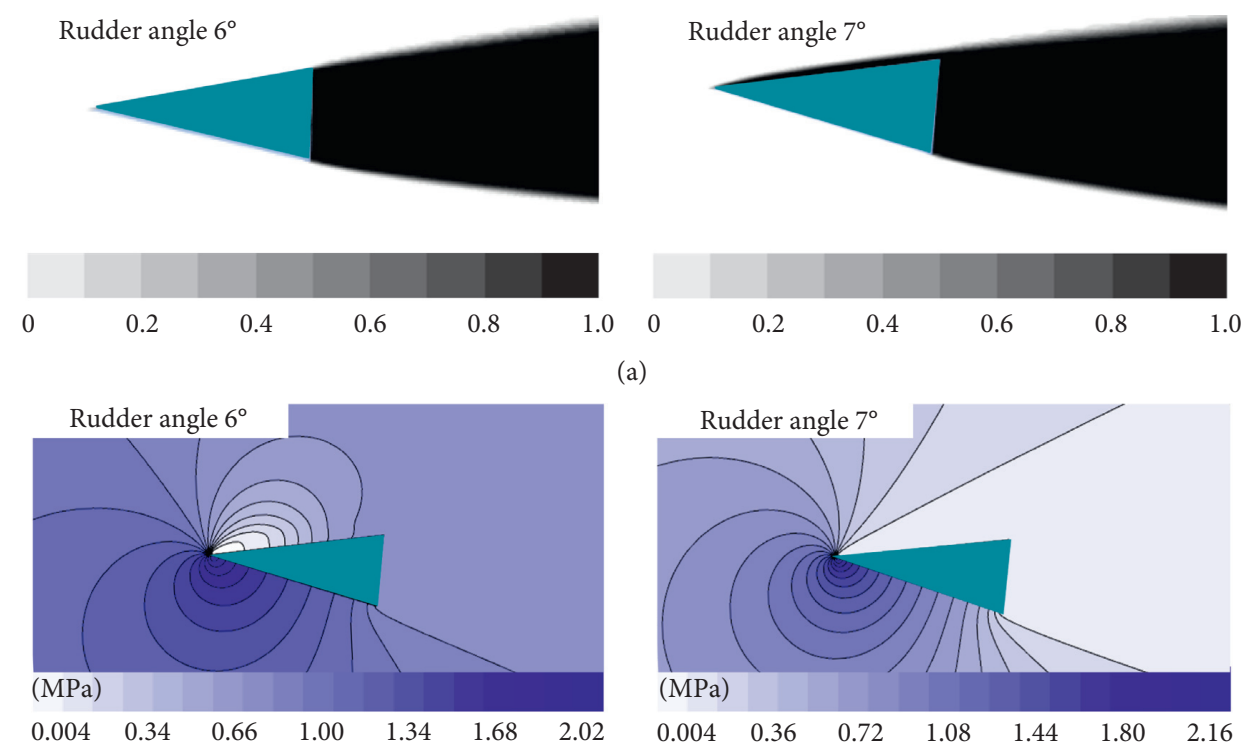

(b)

FIgURE 24: Comparison of flow regime around SR. (a) Comparison of volume fraction of vapor around SR. (b) Comparison of pressure distribution around SR.

Section 4.2.1. By combining the theoretical and numerical methods, the actual wetted area of the SR can be approximately estimated. Moreover, during the dynamic motion of the HSSVs, the vehicle axis is not always coincident with the supercavity axis. The wetted conditions and the lift acting point of a couple of vertical or horizontal SRs are different. Then, an unavoidable rolling moment acts at the HSSV when the couple of SRs operate simultaneously. However, the BRs locate before the beginning of the supercavity, and the wetted conditions and the lift acting point of a couple of vertical or horizontal BRs remain the same. Then, the above challenges cannot be overcome for the HSSV using the BR layout scheme.

\section{Conclusions}

In this paper, the VOF multiphase model is employed to calculate the supercavitation flow field. The influence of the BR and SR on the supercavity shape and their respective hydrodynamic characteristic are compared. Key findings are as follows:

(1) The BR is able to generate more lift force and drag force than the SR under the same wetted area and rudder angle. The lift coefficient of the BR increases continuously with the increasing of the rudder angle, but the lift coefficient of the SR is a piecewise function and increases in $0^{\circ}-6^{\circ}$ and $7^{\circ}-12^{\circ}$, respectively.

(2) The BR results in slight enlargement of the maximum diameter of the supercavity and a considerable deformation at the forepart of the supercavity, and the deformation behind the middle section is limited. The SR provides a tiny influence on the supercavity before it, but a concave deformation is observed around the SR. The concave deformation increases the wetted area of the SR by approximately $29.4 \%$.

(3) For the BR, the wetted area remains unchanged, and the rudder angle needed to provide the desired lift force is unique. For the SR, the wetted area is variable and is influenced by the multifactors; some uncertainties exist in predicting the lift force.

\section{Data Availability}

The data used to support the findings of this study are available from the corresponding author upon request.

\section{Conflicts of Interest}

The authors declare that they have no conflicts of interest.

\section{Acknowledgments}

The project was supported by the National Natural Science Foundation of China (Grants nos. 51909218 and 51679202), China Postdoctoral Science Foundation (Grant no. 2019M653747), and Natural Science Basic Research Plan in Shaanxi Province of China (Program no. 2019JQ-225). The authors would like to thank them for the sponsorship.

\section{References}

[1] Y. N. Savchenko, "Supercavitation-problems and perspectives," in Proceedings of the CAV: 4th International Symposium on Cavitation, pp. 1-8, Pasadena, CA, USA, June 2001.

[2] Y. N. Savchenko, Y. D. Vlasenko, and V. N. Semenenko, "Experimental studies of high-speed cavitated flows," International Journal of Fluid Mechanics Research, vol. 26, no. 3, pp. 365-374, 1999. 
[3] X. Mao and Q. Wang, "Delay-dependent control design for a time-delay supercavitating vehicle model," Journal of $\mathrm{Vi}$ bration and Control, vol. 17, no. 3, pp. 431-448, 2011.

[4] V. Serebryakov, I. Kirschner, and G. Schnerr, "High speed motion in water with supercavitation for sub-, trans-, supersonic mach numbers," in Proceedings of the 7th International Symposium on Cavitation, pp. 1-18, Ann Arbor, MI, USA, August 2009.

[5] D. R. Stinebring, R. B. Cook, J. E. Dzielski et al., "High-speed supercavitating vehicles," in Proceedings of the AIAA Guidance, Navigation, and Control Conference and Exhibit Keystone, vol. 6441, pp. 1-8, Keystone CO, USA,, August 2006.

[6] L. G. Loytsyansky, "About change of body drag by filling the boundary layer by liquid with other physical constants," Journal of Priklaladnaya Matematika I Mehanika, vol. 6, pp. 114-125, 1942, in Russian.

[7] G. V. Logvinovich, "Some problems in planing surfaces," Trudy TsAGI, vol. 1980, pp. 3-12, 2052.

[8] V. N. Semenenko, "Artificial supercavitation. physics and calculation," vol. 11, pp. 1-34, Von Karman Institute for Fluid Dynamics, Brussels, Belgium, 2001, RTO AVT Lecture Series.

[9] A. Karn, R. E. A. Arndt, and J. Hong, "An experimental investigation into supercavity closure mechanisms," Journal of Fluid Mechanics, vol. 789, pp. 259-284, 2016.

[10] X. Yuan and T. Xing, "Hydrodynamic characteristics of a supercavitating vehicle's aft body," Ocean Engineering, vol. 114, pp. 37-46, 2016.

[11] A. May, "Water entry and the cavity-running behaviour of missiles," Report Number: 75-2, Final Technical Report NAVSEA Hydroballistics Advisory Committee, Silver Spring, MD, USA, 1975.

[12] H. Mokhtarzadeh, G. Balas, and R. Arndt, "Effect of cavitator on supercavitating vehicle dynamics," IEEE Journal of Oceanic Engineering, vol. 37, no. 2, pp. 156-165, 2012.

[13] V. Nguyen, Dynamics and Control of Non-smooth Systems with Applications to Supercavitating Vehicles, pp. 1-14, University of Maryland, College Park, MD, USA, 2011.

[14] V. Nguyen, M. A. Hassouneh, B. Balachandran et al., "Nonsteady planning and advection delay effects on the dynamics and control of supercavitating vehicles," in Proceedings of the ASME 2011 International Mechanical Engineering Congress \& Exposition, pp. 1-7, Denver, CO, USA, November 2011.

[15] D. Sanabria and R. Arndt, "Robust control of a small-scale supercavitating vehicle: from modeling to testing," Ocean Engineering, vol. 160, pp. 412-424, 2018.

[16] M. Mirzaei, M. M. Alishahi, and M. Eghtesad, "High-speed underwater projectiles modeling: a new empirical approach," Journal of the Brazilian Society of Mechanical Sciences and Engineering, vol. 37, no. 2, pp. 613-626, 2015.

[17] A. D. Vasin and E. V. Paryshev, "Immersion of a cylinder in a fluid through a cylindrical free surface," Fluid Dynamics, vol. 36, no. 2, pp. 169-177, 2001.

[18] T. Yen, M. Morabito, L. Imas et al., "Investigation of cylinder planning on a flat free surface," in Proceedings of the 11th International Conference on Fast Sea Transportation, pp. 396-403, Honolulu, HI, USA, September 2011.

[19] V. Nguyen and B. Balachandran, "Supercavitating vehicles with noncylindrical, nonsymmetric cavities: dynamics and instabilities," Journal of Computational and Nonlinear Dynamics, vol. 6, no. 4, pp. 1-11, 2011.

[20] D. Li, K. Luo, C. Huang et al., "Dynamics model and control of high-speed supercavitating vehicles incorporated with time delay," International Journal of Nonlinear Sciences and Numerical Simulation, vol. 15, no. 3-4, pp. 221-230, 2014.
[21] D. Li, Q. Liu, K. Qin, C. Huang, K. Luo, and J. Dang, “Classical control of underwater supercavitating vehicles via variable splitting method," Ships and Offshore Structures, vol. 14, no. 7, pp. 765-776, 2019.

[22] K. Luo, D. Li, K. Qin et al., "Hydrodynamic layout of strongly maneuvering underwater supercavitating vehicle," Journal of Traffic and Transportation Engineering, vol. 10, no. 4, pp. 45-57, 2010.

[23] R. Kuklinski and A. Fredette, "Experimental studies in the control of cavitating bodies," in Proceedings of the AIAA Guidance, Navigation, and Control Conference and Exhibit, pp. 1-17, Keystone, CO, USA, August 2006.

[24] C. Huang, J. Dang, K. Luo et al., "Numerical simulation and water tunnel experiment on the stalling characteristics of wedge rudders," Journal of Harbin Institute of Technology, vol. 10, no. 48, pp. 125-130, 2016.

[25] M. A. Gordon, Experimental Characterization of Supercavitating Fins Piercing a Ventilated Cavity Boundary, pp. 19-38, Stevens Institute of Technology, Hoboken, NJ, USA, 2012.

[26] L. G. Guzevsky, "Numerical analysis of cavitational flows," Sub Division of Soviet Academy of Sciences International Journal of Thermophysics Preprint, vol. 40, 1979.

[27] K.-p. Yu, G. Zhang, J.-j. Zhou, W. Zou, and Z.-w. Li, "Numerical study of the pitching motions of supercavitating vehicles," Journal of Hydrodynamics Ser. B, vol. 24, no. 6, pp. 951-958, 2012.

[28] G. H. Schnerr and J. Sauer, "Physical and numerical modeling of unsteady cavitation dynamics," in Proceedings of the Fourth International Conference on Multiphase Flow, p. 1, New Orleans, LA, USA, 2001.

[29] C. Huang, K. Luo, J. Dang et al., "Influence of flow field's radial dimension on natural supercavity," Journal of Northwestern Polytechnical University, vol. 33, no. 6, pp. 936-941, 2015.

[30] C. Huang, K. Luo, J. Dang et al., "Spatial kinetics model of supercavitating vehicles reflecting conic-lsike oscillation," Mathematical Problems in Engineering, vol. 2017, Article ID 3671618, 12 pages, 2017. 